Department of Policy and Planning Sciences

\author{
Discussion Paper Series
}

No.1343

On the operation of multiple matching markets

by

Hidekazu ANNO and Morimitsu KURINO

Mar 2016

UNIVERSITY OF TSUKUBA

Tsukuba, Ibaraki 305-8573 JAPAN 


\title{
On the operation of multiple matching markets *
}

\author{
Hidekazu Anno $^{\dagger} \quad$ Morimitsu Kurino ${ }^{\ddagger}$
}

June 19, 2015

\begin{abstract}
We provide a new perspective on how to operate matching markets when there are many types of markets. Our finding is that the market-wise adaptation of strategy-proof and nonwasteful rules yields a strategy-proof rule with the following efficiency property: no strategyproof rule Pareto-dominates the rule. Such rules are abundant as they include the market-wise use of the well-known priority-based rules of the top trading cycles and the deferred acceptance. This result gives theoretical support to the independent operation of markets observed in real-life markets as well as our practice in Market Design that separately treats each market for its design.
\end{abstract}

Journal of Economic Literature Classification Numbers : C78, D47, D71.

Keywords: Market design; Strategy-proofness; Second-best incentive compatibility; Top trading cycles rules; Deferred acceptance rules.

${ }^{*}$ We would like to thank Takashi Akahoshi, Masaki Aoyagi, Umut Dur, Aytek Erdil, Onur Kesten, Fuhito Kojima, Eiichi Miyagawa, Hervé Moulin, Hiroo Sasaki, Norov Tumennasan, Utku Ünver, Zaifu Yang, and Bumin Yenmez for helpful discussions. We would also like to thank seminar participants at York, Waseda, the 12th meeting of the Society for Social Choice and Welfare at Boston College, the 14th SAET conference at Waseda, and the workshop "Designing Matching Markets" at the WZB Berlin for useful comments. The paper was circulated as "Second-best incentive compatible allocation rules for multiple-type indivisible objects." Hidekazu Anno greatfully acknowledges the Waseda University Grant for Special Research Projects (Project number: 2014B-146) and JSPS KAKENHI Grant Number 24243037. Morimitsu Kurino acknowledges financial support from JSPS KAKENHI Grant number 15H03327, and is thankful to Koichi Takase and the Faculty of Commerce at Waseda University for their hospitality when he was a visitor there during the initial stage of this work. All remaining errors are our own.

${ }^{\dagger}$ Graduate School of Fundamental Science and Engineering, Waseda University, Japan; e-mail: annohidekazu@gmail.com

${ }^{\ddagger}$ Faculty of Engineering, Information and Systems, University of Tsukuba, 1-1-1 Tennodai, Tsukuba, Ibaraki 305-8573, Japan; e-mail: kurino@sk.tsukuba.ac.jp 


\section{Introduction}

We have recently witnessed economists' discovery and design of real-life matching markets such as school choice (Abdulkadiroğlu and Sönmez, 2003) and organ exchanges (Roth, Sönmez, and Ünver, 2004; Ergin, Sönmez, and Ünver, 2014). In such a market one type of indivisible good, or object for short, is allocated to agents who demand one unit when monetary transfers are not allowed (Shapley and Scarf, 1974; Hylland and Zeckhauser, 1979).

We often deal with multiple types of objects at the same time - what we call a multiple-type (matching) market. People simultaneously participate in multiple markets, each of which deals with a different type of commodity. Examples are abundant: Many families live in public housing and have children who go to school - one type of object is public housing and another is a seat in a school; ${ }^{1}$ Teaching courses and administrative tasks are assigned to faculty members in universities where one type of object is courses and another is administrative tasks; About $80 \%$ of pancreas patients in Japan also need a kidney transplant due to chronic kidney disease where one type of object is the pancreas and another the kidney. ${ }^{2}$ Furthermore, a dynamic matching market is a special class of multiple-type markets where people dynamically participate in one market multiple times (Kurino, 2014). This is because the objects consumed in two distinct dates are considered to be different object types. For example, college students take part in the annual assignment of dormitory rooms; Families with several children participate in a school choice program or a daycare assignment program at different times when each of their children enters the school (Dur, 2012; Kennes, Monte, and Tumennasan, 2014); Teachers in public schools move to different schools through annual assignment procedures (Bloch and Cantala, 2013).

In this paper, we focus on a multiple-type market. Each agent has a separable preference, where a preference over bundles is separable if there is a list of preferences over each single-type market such that if two bundles $x$ and $y$ are different in only $t$-th type, then the evaluation between $x$ and $y$ coincides with the evaluation of $t$-th types $x^{t}$ and $y^{t}$ according to the preference over the $t$-th market objects. ${ }^{3}$

One important observation is that in most cases, if not all, assignment procedures for different types of objects are operated by different government authorities, and each procedure is independent from the other. ${ }^{4}$ Corresponding to this practice in real-life markets, the Market Design literature treats each market independently and then considers the design of stratetgy-proof and

\footnotetext{
${ }^{1}$ The percentage of the population living in public housing is about 10 to 35 in many countries. For example, Whitehead and Scanlon (2007) report that the percentages in European countries around 2000 are 35 in the Netherlands, 25 in Austria, 21 in Denmark, 20 in Sweden, 18 in England, 17 in France, 8 in Ireland, 6 in Germany, and 4 in Hungary. Moreover, the percentages are 30 in Hong Kong and 9.9 in Japan (The webpages are http://www.housingauthority.gov.hk/en/public-housing/index.html and http://www.stat.go.jp/data/jyutaku/2008/10_3.htm. The webs were accessed on December 18, 2013.) This suggests that about the same percentage of families with children would participate in two types of markets - public housing assignment and school choice programs.

${ }^{2}$ For example see the Handbook of The Japan Society for Transplantation (2013).

${ }^{3} \mathrm{~A}$ model with single-type objects is a special case of ours. Although our main focus is a multiple-type model, all of our results remain true in single-type models.

${ }^{4}$ For example, in the City of Boston, the school choice assignment is operated by Boston Public Schools, while the public housing assignment is controlled by the Boston Housing Authority.
} 
Table 1: An example of inefficiency

\begin{tabular}{|c||c|c|l|c||c|c|}
\hline \multicolumn{3}{|c|}{ Agent 1 } & & \multicolumn{3}{|c|}{ Agent 2 } \\
\hline$R_{1}$ & $R_{1}^{1}$ & $R_{1}^{2}$ & & $R_{2}$ & $R_{2}^{1}$ & $R_{2}^{2}$ \\
\hline$(a, c)$ & $\underline{a}$ & $c$ & & $(a, c)$ & $a$ & $\underline{c}$ \\
$(b, c)$ & $b$ & $\underline{d}$ & & $(a, d)$ & $\underline{b}$ & $d$ \\
$\frac{(a, d)}{(b, d)}$ & & & & $\frac{(b, c)}{(b, d)}$ & & \\
$\vdots$ & & & & $\vdots$ & & \\
\hline
\end{tabular}

Note: Agent $i$ 's preference for bundles is denoted by $R_{i}$ while that for type- $t$ objects is by $R_{i}^{t}$. For example, agent 1 prefers bundle $(a, c)$ to $(b, c)$ to $(a, d), \ldots$, and she prefers $a$ to $b$ for type 1 while she does $c$ to $d$.

Pareto efficient (or at least non-wasteful) rules for only one type of market. ${ }^{5}$ However, such independent operation of different markets leads to inefficiency when we take all types of markets into account.

To illustrate this point, suppose that there are two agents and two types of objects. We have two objects $a, b$ for type $t=1$ market, and two objects $c, d$ for type $t=2$ market. Preferences of agents 1 and 2 are given by Table 1 . Now, let us consider the underlined allocation in this table where agent 1 receives $(a, d)$ and agent 2 gets $(b, c)$. In other words, for type $t=1$ the allocation prescribes $(1,2 ; a, b)$, while for type $t=2$ it does $(1,2 ; d, c)$. Note that within each type market the allocations $(1,2 ; a, b)$ and $(1,2 ; d, c)$ are Pareto efficient. However, by swapping their bundles both agents are made better off. This means that the original allocation is not Pareto efficient, although its allocation for each type is Pareto efficient. One lesson from this example is that an allocation procedure that uses a Pareto efficient rule for each type might result in an inefficient allocation. ${ }^{6}$

One of the most serious difficulties pertaining to the multiple-type market is that the combination of strategy-proofness and Pareto efficiency essentially results in a serial dictatorship rule in which each agent chooses her assignment one by one according to an exogenously fixed priority order (Monte and Tumennasan, 2013). ${ }^{7}$ However, such a rule is against the independent operation of markets in real life and is extremely unfair. ${ }^{8}$

With this difficulty, we take a natural research direction of looking for a plausible rule by

\footnotetext{
${ }^{5}$ Under a non-wasteful allocation (Balinski and Sönmez, 1999), if some object is preferred to the assigned one for an agent, it is fully assigned up to its quota. Thus, non-wastefulness is an efficiency axiom weaker than Pareto efficiency. Note that non-wastefulness is defined only for single-type markets. The lack of a natural second-best efficiency notion in a multiple-type market is another motivation of the current paper.

${ }^{6}$ More specifically, this is a Pareto inefficient allocation although it is reached by the market-wise serial dictatorship rule which is strategy-proof and Pareto efficient in each type market. Agent 1 is the dictator of the first market while agent 2 is the dictator of the second market.

${ }^{7}$ Rigorously speaking, Monte and Tumennasan's (2013) model is not a special case of ours due to the difference in preference domains (See Assumption 2 and footnote 17). They prove that if a rule is strategy-proof, Pareto efficient and non-bossy, then it is a sequential dictatorship rule that is a variant of the serial dictatorship rule.

${ }^{8}$ Consider the environment with homogeneous preferences of agents and unit quotas of objects. In the "full" serial dictatorship (SD) rule, the highest-priority agent receives her favorite objects from all types, and is envied by all agents in each type. However, we can mitigate the unfairness with a "market-wise" serial dictatorship (SD) rule where a priority is defined for each type, and the highest-priority agent in a type is different from those in the other markets. In this case for each agent there is only one type market for which she is envied by all agents. Although it is Pareto inefficient, the market-wise SD rule to a large extent remedies the unfair feature of the full SD rule.
} 
relaxing Pareto efficiency while keeping strategy-proofness. That is, we search for "second-best (efficient) incentive compatible" rules. To be precise, we call a rule second-best incentive compatible if the rule is itself strategy-proof, and is not Pareto-dominated by any other strategy-proof rule. It is a quite natural second-best efficiency concept as far as we are concerned with strategy-proofness since the notion is a straightforward adaptation of the Pareto criterion for the class of strategy-proof rules. This paper provides a simple and practical sufficient condition for the second-best incentive compatibility.

To state our main result, it is worth noting that there is a very simple way to construct a strategy-proof rule in a multiple-type market. Namely, we first consider an independent rule which applies a single-type rule in each market, and then consider only strategy-proof single-type rules for an independent rule. This is made possible due to the separability of preferences. Based on this, our main result (Theorem 1) is the following: An independent rule obtained by the market-wise application of strategy-proof and non-wasteful single-type rules is second-best incentive compatible.

Now, let us explain the economic implications of Theorem 1. Inspired by Gale's top trading cycles (Shapley and Scarf, 1974) and Gale and Shapley's (1962) deferred acceptance algorithm, the Market Design literature has uncovered strategy-proof and non-wasteful rules for various singletype matching problems such as school choice problems, on-campus housing assignments and kidney exchange problems for patients (See section 5). However, as we have already seen, even if we successfully design such a strategy-proof and Pareto efficient rule for each single-type market, the rules may collectively fail to be Pareto efficient as an allocation procedure for a multiple-type market. Theorem 1 guarantees that the practices of designing such a rule will lead to second-best incentive compatibility - the impossibility of being Pareto-improved without sacrificing strategyproofness. Moreover, we have a rich class of second-best incentive compatible rules, since most of prominent single-type rules, if not all, proposed in the literature are strategy-proof and nonwasteful. The positive result and the richness of rules are in marked contrast to requiring the "firstbest incentive compatibility" (namely, the combination of strategy-proofness and Pareto efficiency) which often results in an extremely unfair rule of serial dictatorship in our multiple-type setting. That is, the efficiency loss in these rules is an inevitable cost of recovering the fairness and operating markets independently as long as we preserve strategy-proofness.

In sum Theorem 1 provides us with a new perspective for Matching Market Design - the theoretical support for the independent operation and design of matching markets with a strategy-proof and non-wasteful rule for each type. ${ }^{9}$ The support is even stronger if we note that a decentralized trade from an arbitrary allocation would not work in a multiple-type market as the core may be empty (Konishi, Quint, and Wako, 2001). ${ }^{10}$

\footnotetext{
${ }^{9}$ This is reminiscent of the first welfare theorem in the standard competitive equilibrium theory which guarantees that if each independent individual "rationally" behaves in markets, the resulting allocation is efficient. Likewise, in our multiple-type market, if each independent designer uses strategy-proof and (at least) non-wasteful rule in each market, the resulting rule is second-best efficient for the whole market.

${ }^{10}$ We mean that a decentralized trade is one where mutually beneficial trades would be made until there are no such trades. The core is defined to be an allocation from which there is no further mutually beneficial trade. Note that in a single-type market the core is singleton and thus the procedure works well for reaching the unique allocation in the core (Shapley and Scarf, 1974; Roth and Postlewaite, 1977).
} 


\section{$1.1 \quad$ Related literature}

The most basic matching model is a house allocation problem (Shapley and Scarf, 1974; Hylland and Zeckhauser, 1979) where each agent demands one unit of an object. In addition to real-life applications mentioned in the Introduction there are on-campus housing assignments for college students (Abdulkadiroğlu and Sönmez, 1999) and cadet-branch matching problem in the United States Military Academy (Sönmez and Switzer, 2013). See Sönmez and Ünver (2011) for their comprehensive knowledge on Matching Market Design.

In contrast to the literature on the above basic problems, there are few papers on multipletype markets: Moulin (1995) introduces the model with an initial endowment, referred to as multiple-type housing market, or generalized Shapley-Scarf housing market. Konishi, Quint, and Wako (2001) show that the core might be empty in a multiple-type housing market. Moreover, they show that no rule is strategy-proof, Pareto efficient, and individually rational. Finally, Klaus (2008) shows that the market-wise core mechanism satisfies the second-best incentive compatibility. Similar to our paper, Monte and Tumennasan (2013) investigate a multiple-type markets without endowments, and show that the combination of strategy-proofness, Pareto efficiency, and nonbossiness implies the sequential dictatorship.

There is a similar but different problem of multiple assignment where people demand multiple units of objects though there is no type of objects. Thus the multiple assignment problem is less restrictive than a multiple-type market because objects are exogenously divided into different groups in a multiple-type market. Pápai (2001) and Hatfield (2009) show that the combination of strategy-proofness, Pareto efficiency, and non-bossiness results in a sequential dictatorship rule. Klaus and Miyagawa (2001) also show a dictatorship result under solidarity and consistency when the population can vary. Moreover, Sönmez and Ünver (2010); Budish and Cantillon (2012) examine course allocations at business schools.

The separability of preference is one of the key features in the theory where each agent consumes multi unit of objects. The separability is assumed for the many-side agents in a many-to-one two-sided matching market, agents in multiple-type markets, and those in multiple assignment problems. Moreover, the studies of social choice functions on product spaces work on the separable preference domain (e.g., Barberà, Sonnenschein, and Zhou 1991; Le Breton and Sen 1999; Ju 2003; Barberà, Massó, and Neme 2005). On the other hand, the study under interdependence or nonseparability of preferences has just started in matching models. In particular, Dur (2012) and Dur and Wiseman (2015) consider a problem to allocate a number of school seats to the families with siblings and neighbors. They consider a specific non-separable preference domain where each family or neighbors prefer to receive the same school for their children. Thus, it is an important issue to select a realistic interdependence of preferences corresponding to the multiple-type market under consideration. Although we focus on separable preferences in this paper, we believe that our results work as a benchmark and bring insights for non-separable preference domains.

With the separable preferences, an independent rule, which is our focus in our paper, is welldefined where the entire rule consists independently of type-market rules. A few papers deal with dependent rules. For example, in his introduced house allocation problem with the overlapping 
generations structure, ${ }^{11}$ Kurino (2014) considers a dependent rule under separable preferences where in each period the previously assigned object becomes an endowment and thus affects the current rule through endowments. Kennes, Monte, and Tumennasan (2014) extend Kurino's (2014) model to a school choice problem under separable preferences, and focus on dependent rules where in each period the previously assigned object affects the current priority and thus type-rule. ${ }^{12}$

The taxation literature has the tradition to consider second-best efficient allocations. ${ }^{13}$ As is defined formally in Section 4, the second-best efficiency concept in this paper concerns about the efficiency of an allocation rule under the constraint of strategy-proofness. To the best of our knowledge, a variant of second-best incentive compatibility is first studied in Sasaki (2003) in the context of divisible resource allocation problems with multi-dimensional single-peaked preferences. The second-best incentive compatibility in the current paper is studied in Anno (2008); Anno and Sasaki (2013) for the same model as Sasaki (2003). Moreover, Klaus (2008) investigates the concept in a multiple-type housing market, while Abdulkadiroğlu, Pathak, and Roth (2009), Kesten and Kurino (2013), Erdil (2014), and Fragiadakis and Troyan (2013) study it in single-type problems.

\section{Model and Axioms}

\subsection{Multiple-type markets}

We introduce a general model of multiple-type markets where an agent is interested in being assigned multiple types of objects when monetary transfers are not allowed. A multiple-type market is a list $\left(N, T,\left(T_{i}\right)_{i \in N},\left(X^{t}\right)_{t \in T}, q,\left(R_{i}\right)_{i \in N}\right): N:=\{1, \ldots, n\}$ is a finite set of agents with $|N| \geq 2$, while $T$ is a finite set of types of objects (i.e., indivisible goods). We identify $T$ with $\{1, \cdots,|T|\}$. If $|T|=1$, we call a market single type. For each type $t \in T$, objects of type- $t$ are available. Let $X^{t}$ be a finite set of type-t objects with $\left|X^{t}\right| \geq 2$. For each type $t \in T, q\left(x^{t}\right)$ is the quota of type- $t$ object $x^{t} \in X^{t}$. That is, $q$ is a function from $\cup_{t \in T} X^{t}$ to $\mathbb{Z}_{++}$and $q\left(x^{t}\right)$ indicates the number of identical type-t object $x^{t}$. Each agent $i \in N$ is interested in at least one type of objects, and consumes one object from each type in which she is interested. Let $T_{i} \in 2^{T} \backslash\{\emptyset\}$ be the set of types in which agent $i$ is interested (See Table 2). Moreover, for each type $t \in T$, let $N^{t}:=\left\{i \in N \mid t \in T_{i}\right\}$ be the set of agents who are interested in type- $t$ objects. We assume that for each $t \in T$, at least one agent is interested in the type- $t$, i.e., $N^{t} \neq \emptyset$. Throughout the paper we maintain the following assumption. ${ }^{14}$

Assumption 1 (Existence of outside option). For each type $t \in T$, there is the type- $t$ outside option, denoted by $o^{t}$, in $X^{t}$ which satisfies $q\left(o^{t}\right) \geq\left|N^{t}\right|$.

In words, the assumption guarantees that every agent has the right to withdraw from the market. The condition $\left(q\left(o^{t}\right) \geq\left|N^{t}\right|\right)$ means that the outside option $o^{t}$ is sufficiently available

\footnotetext{
${ }^{11}$ In this interpretation, the set of types is viewed as the set of periods. Since we assume that the set of types is finite, our model can accommodate a dynamic matching model with a finite horizon.

${ }^{12}$ Pereyra (2013) also extends Kurino's (2014) to a school choice problem and then considers independent rules under separable preferences.

${ }^{13}$ Hammond (2000) provides a good summary of the literature.

${ }^{14}$ The only exception is the paragraph right before Corollary 3. There we do not assume Assumption 1.
} 
Table 2: An example of a multiple-type market

\begin{tabular}{c|c|c|c|c} 
& Type 1 & Type 2 & Type 3 & \\
\hline Agent 1 & $\sqrt{ }$ & & & $T_{1}=\{1\}$ \\
\hline Agent 2 & $\sqrt{ }$ & $\sqrt{ }$ & & $T_{2}=\{1,2\}$ \\
\hline Agent 3 & & $\sqrt{ }$ & $\sqrt{ }$ & $T_{3}=\{2,3\}$ \\
\hline & $N^{1}=\{1,2\}$ & $N^{2}=\{2,3\}$ & $N^{3}=\{3\}$ &
\end{tabular}

Note: In this example, there are three agents and three types. The symbol $\sqrt{ }$ indicates which agent is interested in what types of objects. For example, agent 2 is interested in both types 1 and 2 .

so that it can be consumed by all agents interested in the type- $t$ market at the same time. We interpret it as the artificial object that represents the option to withdraw. We discuss in the conclusion how this assumption affects our main result.

Finally, we describe the preferences of agents. Since each agent $i \in N$ consumes one object from each type in $T_{i}$, her consumption space is $X_{i}:=\prod_{t \in T_{i}} X^{t}$. An element of $X_{i}$ is called a bundle, generically denoted by $x_{i}=\left(x_{i}^{t}\right)_{t \in T_{i}} \in X_{i}$. For convenience and clarity, we introduce some notations: For a finite set $Y$, let $\mathcal{R}(Y)$ be the set of all complete and transitive binary relations on $Y$, and $\mathcal{P}(Y)$ the set of all complete, transitive, and anti-symmetric binary relations on $Y{ }^{15}$ Each agent $i \in N$ is equipped with a preference relation $R_{i}$ on $X_{i}$, i.e., $R_{i} \in \mathcal{R}\left(X_{i}\right)$. For each $R_{i} \in \mathcal{R}\left(X_{i}\right), P_{i}$ and $I_{i}$ denote the asymmetric and symmetric parts of $R_{i}$. We denote $R:=$ $\left(R_{i}\right)_{i \in N} \in \prod_{i \in N} \mathcal{R}\left(X_{i}\right)$ and call it a profile. For each profile $R=\left(R_{1}, \cdots, R_{n}\right) \in \prod_{i \in N} \mathcal{R}\left(X_{i}\right)$, and each $i \in N$, the subprofile obtained by removing $i$ 's preference is denoted by $R_{-i}$; that is, $R_{-i}:=$ $\left(R_{1}, \cdots, R_{i-1}, R_{i+1}, \cdots, R_{n}\right)$. It is convenient to write the profile $\left(R_{1}, \cdots, R_{i-1}, R_{i}^{\prime}, R_{i+1}, \cdots, R_{n}\right)$ as $\left(R_{i}^{\prime} ; R_{-i}\right)$.

Now, we introduce two classes of preferences. First, a preference $R_{i} \in \mathcal{R}\left(X_{i}\right)$ is separable if for each $t \in T_{i}$ there is a preference $R_{i}^{t} \in \mathcal{P}\left(X^{t}\right)$ on type- $t$ objects such that for each pair of bundles $\left\{x_{i}, x_{i}^{\prime}\right\} \subseteq X_{i}$,

$$
\text { [ for each } \left.t \in T_{i}, x_{i}^{t} R_{i}^{t} x_{i}^{\prime t}\right] \Rightarrow x_{i} R_{i} x_{i}^{\prime} \text {. }
$$

We denote by $\mathcal{R}_{\text {sep }}\left(X_{i}\right)$ the set of all separable preferences on $X_{i}$, and call $R_{i}^{t}$ a type- $t$ preference. Let $\mathcal{P}_{\text {sep }}\left(X_{i}\right)$ be the set of all separable strict preferences on $X_{i}$, i.e., $\mathcal{P}_{\text {sep }}\left(X_{i}\right)=\mathcal{R}_{\text {sep }}\left(X_{i}\right) \cap \mathcal{P}\left(X_{i}\right)$.

To introduce the second class of preferences, for each $t \in T$, let $\mathcal{U}^{t}$ be the set of strict utility functions on $X^{t}$. That is, $u_{i}^{t}: X^{t} \rightarrow \mathbb{R}$ belongs to $\mathcal{U}^{t}$ if $u_{i}^{t}$ is injective. A preference $R_{i} \in \mathcal{R}\left(X_{i}\right)$ is additively separable if for each $t \in T_{i}$ there is a utility function $u_{i}^{t} \in \mathcal{U}^{t}$ on type- $t$ objects such that for each pair of bundles $\left\{x_{i}, x_{i}^{\prime}\right\} \subseteq X_{i}$,

$$
x_{i} R_{i} x_{i}^{\prime} \Leftrightarrow \sum_{t \in T_{i}} u_{i}^{t}\left(x_{i}^{t}\right) \geq \sum_{t \in T_{i}} u_{i}^{t}\left(x_{i}^{\prime t}\right)
$$

We denote by $\mathcal{R}_{a d d}\left(X_{i}\right)$ the set of all additively separable preferences on $X_{i}$, and by $\mathcal{P}_{\text {add }}\left(X_{i}\right)$ the set of all additively separable strict preferences on $X_{i}$, i.e., $\mathcal{P}_{\text {add }}\left(X_{i}\right)=\mathcal{R}_{\text {add }}\left(X_{i}\right) \cap \mathcal{P}\left(X_{i}\right)$. The

\footnotetext{
${ }^{15} \mathrm{~A}$ binary relation $\geq$ is complete if for each $\left\{y, y^{\prime}\right\} \subseteq Y, y \geq y^{\prime}$ or $y^{\prime} \geq y$. A binary relation $\geq$ is transitive if for each $\left\{y, y^{\prime}, y^{\prime \prime}\right\} \subseteq Y, y \geq y^{\prime}$ and $y^{\prime} \geq y^{\prime \prime}$ imply $y \geq y^{\prime \prime}$. A binary relation $\geq$ is anti-symmetric if for each $\left\{y, y^{\prime}\right\} \subseteq Y, y \geq y^{\prime}$ and $y^{\prime} \geq y$ imply $y=y^{\prime}$.
} 
next remark immediately follows from the definitions.

Remark 1. Let $i \in N$.

1. For each $R_{i} \in \mathcal{P}_{\text {sep }}\left(X_{i}\right) \cup \mathcal{R}_{a d d}\left(X_{i}\right)$, the list of corresponding type- $t$ preferences $\left(R_{i}^{t}\right)_{t \in T_{i}}$ is unique.

2. We have the following relations among the classes of preferences. ${ }^{16}$

$$
\begin{array}{cccc}
\mathcal{P}_{\text {add }}\left(X_{i}\right) & \subsetneq & \mathcal{P}_{\text {sep }}\left(X_{i}\right) \\
\cap \uparrow & & \cap \uparrow \\
\mathcal{R}_{\text {add }}\left(X_{i}\right) & \subsetneq & \mathcal{R}_{\text {sep }}\left(X_{i}\right)
\end{array}
$$

Let $\mathcal{D}_{i}$ be the set of agent $i$ 's admissible preferences. Let $\mathcal{D}:=\prod_{i \in N} \mathcal{D}_{i}$ be the set of admissible profiles. In the rest of the paper we keep the following assumption.

Assumption 2 (Admissible preferences). For each $i \in N, \mathcal{P}_{\text {add }}\left(X_{i}\right) \subseteq \mathcal{D}_{i} \subseteq \mathcal{P}_{\text {sep }}\left(X_{i}\right) \cup$ $\mathcal{R}_{\text {add }}\left(X_{i}\right)$.

Our domain covers two types of wide ranges of domains: When one type consists of separable strict preferences, it ranges from the additively separable domain to the universal one (See Item 2 in Remark 1, in particular $\left.\mathcal{P}_{\text {add }}\left(X_{i}\right) \subseteq \mathcal{P}_{\text {sep }}\left(X_{i}\right)\right)$. The other type ranges from the strict domain to the weak one when it consists of additively separable preferences (See Item 2 in Remark 1, in particular $\left.\mathcal{P}_{\text {add }}\left(X_{i}\right) \subseteq \mathcal{R}_{\text {add }}\left(X_{i}\right)\right)$. As long as we keep a separability of preferences, ours is the most natural and covers the widest range of domains in the literature. ${ }^{17}$ This completes the description of a multiple-type market $\left(N, T,\left(T_{i}\right)_{i \in N},\left(X^{t}\right)_{t \in T}, q,\left(R_{i}\right)_{i \in N}\right)$. We assume throughout the paper that $N, T,\left(T_{i}\right)_{i \in N},\left(X^{t}\right)_{t \in T}$, and $q$ are fixed.

Let us emphasize that our model allows for agents' partial participation on type markets $\left(T_{i} \subseteq\right.$ $T$ ) and covers the full participation on all type markets (for each $i \in N, T_{i}=T$ ) considered in the literature. $^{18}$

\subsection{Type markets}

Let a multiple-type market $\left(N, T,\left(T_{i}\right)_{i \in N},\left(X^{t}\right)_{t \in T}, q,\left(R_{i}\right)_{i \in N}\right)$ be given. Then, by Assumption 2 and Item 1 in Remark 1 , for each $i \in N$ and each $R_{i} \in \mathcal{D}_{i}$, each of the corresponding type- $t$ preference is unique and strict, and thus denoted by $R_{i}^{t}$. Thus, it makes sense to introduce the type- $t$ market induced from the multiple-type market. The induced type- $t$ market is the singletype market $\left(N^{t}, X^{t},\left.q\right|_{X^{t}},\left(R_{i}^{t}\right)_{i \in N^{t}}\right)$ where each agent $i \in N^{t}$ consumes one type- $t$ object in $X^{t}$; $\left.q\right|_{X^{t}}$ is the restriction of $q$ to $X^{t}$ which indicates the quota $q\left(x^{t}\right)$ of each type-t object $x^{t} \in X^{t}$.

\footnotetext{
${ }^{16}$ Non-trivial statements are $\mathcal{P}_{a d d}\left(X_{i}\right) \neq \mathcal{P}_{\text {sep }}\left(X_{i}\right)$, and $\mathcal{R}_{a d d}\left(X_{i}\right) \neq \mathcal{R}_{\text {sep }}\left(X_{i}\right)$. The proof of the latter can be found on page 43 in Fishburn (1970). The former is proved in Appendix A.

${ }^{17}$ See Konishi, Quint, and Wako (2001); Klaus (2008); Monte and Tumennasan (2013) whose domain is the set of separable strict preferences, $\mathcal{P}_{\text {sep }}\left(X_{i}\right)$. An exception is Kurino (2014) who allows weak preferences, though his dynamic model is slightly different from the multiple-type goods model. Strictly speaking, Monte and Tumennasan's (2013) domain additionally assumes that the bundle consisting of outside options is worst.

${ }^{18}$ See Moulin (1995); Konishi, Quint, and Wako (2001); Klaus (2008); Monte and Tumennasan (2013).
} 
Finally, $R_{i}^{t}$ is the type- $t$ preference of agent $i$ in $\mathcal{P}\left(X^{t}\right)$. We denote $R^{t}:=\left(R_{i}^{t}\right)_{i \in N^{t}} \in \mathcal{P}\left(X^{t}\right)^{N^{t}}$ and call it the type- $t$ profile induced from $\left(R_{i}\right)_{i \in N}$, or just the type profile induced from $\left(R_{i}\right)_{i \in N} .{ }^{19}$ Similarly to the notations of profiles, we use $R_{-i}^{t}=\left(R_{1}^{t}, \cdots, R_{i-1}^{t}, R_{i+1}^{t}, \cdots, R_{n}^{t}\right)$ and $\left(R_{i}^{\prime t} ; R_{-i}^{t}\right)=\left(R_{1}^{t}, \cdots, R_{i-1}^{t}, R_{i}^{\prime t}, R_{i+1}^{t}, \cdots, R_{n}^{t}\right)$.

Note that our single-type market or type market, $\left(N^{t}, X^{t},\left.q\right|_{X^{t}},\left(R_{i}^{t}\right)_{i \in N^{t}}\right)$, is the traditional house allocation problem (Hylland and Zeckhauser, 1979), which can include the following real life matching problems; House allocation with existing tenants, Kidney exchange, School choice. Section 5 provides a description of these markets. See Sönmez and Ünver (2011) for a comprehensive survey on the subject.

\subsection{Rules}

A type- $t$ allocation is a function $a^{t}$ from $N^{t}$ to $X^{t}$ such that each agent $i \in N^{t}$ is assigned type- $t$ object $a_{i}^{t}$, and for each type-t object $x^{t} \in X^{t}$ the number of agents who are assigned $x^{t}$ does not exceed the quota $q\left(x^{t}\right)$, i.e., $\left|\left\{i \in N^{t} \mid a_{i}^{t}=x^{t}\right\}\right| \leq q\left(x^{t}\right)$. Let $\mathcal{A}^{t}$ be the set of all type- $t$ allocations. An allocation, consisting of type allocations, is $a:=\left(a^{1}, \cdots, a^{|T|}\right) \in \prod_{t \in T} \mathcal{A}^{t}$ where for each $t \in T, a^{t}$ is a type- $t$ allocation. Let $\mathcal{A}:=\prod_{t \in T} \mathcal{A}^{t}$ be the set of all allocations. Given $a \in \mathcal{A}$, for each $i \in N$, let $a_{i}$ be the agent $i$ 's bundle at $a$, i.e., $a_{i}:=\left(a_{i}^{t}\right)_{t \in T_{i}}$.

We focus on deterministic rules in this paper. A rule selects an allocation for each profile in a multiple-type market, i.e., it is a function $\varphi: \mathcal{D} \rightarrow \mathcal{A}$. For each $R \in \mathcal{D}, \varphi_{i}(R)$ denotes the agent $i$ 's bundle at $\varphi(R)$, and $\varphi^{t}(R)$ denotes the type- $t$ allocation at $\varphi(R)$. On the other hand, a type-t rule selects a type- $t$ allocation for each type- $t$ profile, i.e., it is a function $\Phi^{t}: \mathcal{P}\left(X^{t}\right)^{N^{t}} \rightarrow \mathcal{A}^{t}$. For each $R^{t} \in \mathcal{P}\left(X^{t}\right)^{N^{t}}, \Phi_{i}^{t}\left(R^{t}\right)$ denotes the agent $i$ 's type- $t$ object at $\Phi^{t}\left(R^{t}\right)$. Note that $\Phi^{t}\left(R^{t}\right)$ depends only on the preferences of $N^{t}$ while $\varphi^{t}(R)$ may depend on the preferences of $N \backslash N^{t}$. In the conclusion we discuss how our main result can be affected for a lottery rule.

Given a list of type rules, $\left(\Phi^{t}\right)_{t \in T}$, we can naturally define a rule for bundles due to separable preferences:

Definition 1. A rule $\varphi$ is independent if there exists a list of type rules $\left(\Phi^{t}\right)_{t \in T}$ such that for each $R \in \mathcal{D}$ and each $t \in T, \varphi^{t}(R)=\Phi^{t}\left(R^{t}\right)$. If such a $\left(\Phi^{t}\right)_{t \in T}$ exists, it is unique. Thus, if a rule $\varphi$ is independent, we denote its corresponding type- $t$ rule by $\Phi^{t}$.

An independent rule, $\varphi$, treats each type market independently and separately in that a type- $t$ allocation under $\varphi$ depends only on type- $t$ preference profiles. Note also that an independent rule is less informationally demanding than a dependent one, because the former only requires type preferences and the latter requires preferences on bundles that contain type preferences.

Finally, we introduce the dominations of rules: An allocation $a \in \mathcal{A}$ (Pareto) dominates an allocation $b \in \mathcal{A}$ at $R \in \mathcal{D}$, written as $a \operatorname{dom}(R) b$, if for each $i \in N, a_{i} R_{i} b_{i}$, and for some $i \in N, a_{i} P_{i} b_{i}$. Similarly, a type-t allocation $a^{t} \in \mathcal{A}^{t}$ dominates a type- $t$ allocation $b^{t} \in \mathcal{A}^{t}$ at $R^{t} \in \mathcal{P}\left(X^{t}\right)^{N^{t}}$, written as $a^{t} \operatorname{dom}\left(R^{t}\right) b^{t}$, if for each $i \in N^{t}, a_{i}^{t} R_{i}^{t} b_{i}^{t}$, and for some $i \in N^{t}$,

\footnotetext{
${ }^{19}$ In the list $\left(N^{t}, X^{t},\left.q\right|_{X^{t}},\left(R_{i}^{t}\right)_{i \in N^{t}}\right)$, for simplicity we omit the type set and the structure of interested types. Note that in the type- $t$ market, the type set is $\{t\}$ and every agent $i \in N^{t}$ is interested in type- $t$.
} 
$a_{i}^{t} P_{i}^{t} b_{i}^{t}$. Now we can define the domination of rules: A rule $\varphi$ dominates another rule $\zeta$, written as $\varphi \operatorname{dom} \zeta$ if for each $R \in \mathcal{D}$ and each $i \in N, \varphi_{i}(R) R_{i} \zeta_{i}(R)$, and for some $R \in \mathcal{D}, \varphi(R)$ dominates $\zeta(R)$ at $R$.

\subsection{Axioms}

We introduce axioms for both rules and type rules.

The first is an incentive compatibility axiom that says no agent can benefit from misreporting her preference. A rule $\varphi$ is strategy-proof if for each $R \in \mathcal{D}$, each $i \in N$ and each $R_{i}^{\prime} \in \mathcal{D}_{i}$, $\varphi_{i}(R) R_{i} \varphi_{i}\left(R_{i}^{\prime} ; R_{-i}\right)$. Similarly, a type- $t$ rule $\Phi^{t}$ is strategy-proof if for each $R^{t} \in \mathcal{P}\left(X^{t}\right)^{N^{t}}$, each $i \in N^{t}$ and each $R_{i}^{\prime t} \in \mathcal{P}\left(X^{t}\right), \Phi_{i}^{t}\left(R^{t}\right) R_{i}^{t} \Phi_{i}^{t}\left(R_{i}^{\prime t} ; R_{-i}^{t}\right)$.

The second is an efficiency axiom that says, for each profile, the selected allocation should not be dominated by any other allocation at the profile. A rule $\varphi$ is Pareto efficient if for each profile $R \in \mathcal{D}$, there is no allocation $a \in \mathcal{A}$ such that $a$ dominates $\varphi(R)$ at $R$. Similarly, a type- $t$ rule $\Phi^{t}$ is Pareto efficient if for each type-t profile $R^{t} \in \mathcal{P}\left(X^{t}\right)^{N^{t}}$, there is no type-t allocation $a^{t} \in \mathcal{A}^{t}$ such that $a^{t}$ dominates $\Phi^{t}\left(R^{t}\right)$ at $R^{t}$.

The third is a weak efficiency axiom defined only for a type rule. A type- $t$ rule $\Phi^{t}$ is nonwasteful (Balinski and Sönmez, 1999) if for each $R^{t} \in \mathcal{P}\left(X^{t}\right)^{N^{t}}$, each $i \in N^{t}$ and each $x^{t} \in X^{t}$, $x^{t} P_{i}^{t} \Phi_{i}^{t}\left(R^{t}\right)$ implies $\left|\left\{j \in N^{t} \mid \Phi_{j}^{t}\left(R^{t}\right)=x^{t}\right\}\right|=q\left(x^{t}\right)$.

The last one is a very weak form of a voluntary participation axiom that says for each profile no agent can be worse off than nothing. A rule $\varphi$ is individually rational if for each $R \in \mathcal{D}$ and each $i \in N, \varphi_{i}(R) R_{i}\left(o^{t}\right)_{t \in T_{i}}$. Moreover, a type- $t$ rule $\Phi^{t}$ is individually rational if for each $R^{t} \in \mathcal{P}\left(X^{t}\right)^{N^{t}}$ and each $i \in N^{t}, \Phi_{i}^{t}\left(R^{t}\right) R_{i}^{t} o^{t}$.

The following results are straightforward from definitions.

Remark 2. Let $\Phi^{t}$ be a type- $t$ rule.

1. If $\Phi^{t}$ is Pareto efficient, then $\Phi^{t}$ is non-wasteful. The converse is not always true.

2. If $\Phi^{t}$ is non-wasteful, then $\Phi^{t}$ is individually rational. The converse is not always true.

Remark 3. Suppose that a rule $\varphi$ is independent.

1. For each $t \in T, \Phi^{t}$ is strategy-proof if and only if $\varphi$ is strategy-proof.

2. If for each $t \in T, \Phi^{t}$ is individually rational, then $\varphi$ is individually rational. The converse is not always true.

3. If $\varphi$ is Pareto efficient, then for each $t \in T, \Phi^{t}$ is Pareto efficient. The convese is not always true.

Note that the example in Introduction is a proof of the latter statement in Item 3 - an independent rule consisting of Pareto efficient type-rules is not Pareto efficient in a multiple-type market. 


\section{Three Classes of Priority-based Rules}

We first introduce a priority profile, and then the three priority-based rules that have played central roles in the literature. The single-type market with priority profiles is called a school choice problem or a priority-based indivisible goods resource allocation problem in the literature.

A priority is defined for each type- $t$ object that orders all agents who are interested in the type- $t$ market and expresses how each agent is treated for the object. Formally, a priority of type- $t$ object $x^{t} \in X^{t}$ is a linear order in $\mathcal{P}\left(N^{t}\right)$, denoted by $\succeq_{x^{t}}^{t}$. We denote $\succeq^{t}:=\left(\succeq_{x^{t}}^{t}\right)_{x^{t} \in X^{t}} \in \mathcal{P}\left(N^{t}\right)^{X^{t}}$ and call it a type- $t$ priority profile. Moreover, we denote $\succeq:=\left(\succeq^{t}\right)_{t \in T} \in \prod_{t \in T} \mathcal{P}\left(N^{t}\right)^{X^{t}}$ a priority profile.

\subsection{Market-wise top trading cycles (TTC) rule}

The top trading cycles (TTC) rule for a single-type market is introduced by Abdulkadiroğlu and Sönmez (2003) who modify Gale's top trading cycles described in Shapley and Scarf (1974). Given a type- $t$ profile $R^{t} \in \mathcal{P}\left(X^{t}\right)^{N^{t}}$, the top trading cycles (TTC) type-rule induced by a type- $t$ priority profile $\succeq^{t}$, denoted by $T T C^{\succeq^{t}}$, selects a type- $t$ allocation as follows:

Step 1: Each agent points to the most favorite object according to her preference and each object points to the agent who has the highest priority for that object. Note that there is at least one cycle. ${ }^{20}$ Each agent in a cycle receives the object she points to and is removed from the market. Each object in a cycle whose quota is one is also removed.

Step $k(\geq 2)$ : Each agent who has not been removed in previous steps points to the most favorite object among the remaining objects according to her preference and each remaining object points to the agent who has the highest priority among the remaining agents for that object. Note that there is at least one cycle. Each agent in a cycle receives the object she points to and is removed from the market. Each object in a cycle is also removed if the number of cycles containing the object formed through $k$ steps is equal to the quota of the object.

The algorithm terminates when no agent remains in the market.

Remark 4. For each type $t \in T$ and each priority profile $\succeq^{t} \in \mathcal{P}\left(N^{t}\right)^{X^{t}}$, the TTC type-rule induced by $\succeq^{t}$ is strategy-proof and Pareto efficient (Abdulkadiroğlu and Sönmez, 2003), and thus is nonwasteful and individually rational by Remark 2.

Definition 2. The market-wise top trading cycles (TTC) rule induced by a priority profile $\succeq=\left(\succeq^{t}\right)$, denoted by $T T C^{\succeq}$, selects its type- $t$ allocation as $T T C^{\succeq^{t}}\left(R^{t}\right)$ that is chosen by the TTC type rule induced by type- $t$ priority profile $\succeq^{t}$.

Remark 5. Note that by Remarks 3 and 4, a market-wise TTC rule is strategy-proof and individually rational. However, the example in Introduction can be used to show that it is not Pareto efficient for the priority profile in Table 3 .

\footnotetext{
${ }^{20} \mathrm{~A}$ cycle is an ordered list of agents and objects $\left(i_{1}, x_{1}, i_{2}, x_{2}, \ldots, i_{m}, x_{m}\right)$ such that $i_{1}$ points to $x_{1}, x_{1}$ points to $i_{2}, i_{2}$ points to $x_{2}, \ldots, i_{m}$ points to $x_{m}$, and $x_{m}$ points to $i_{1}$.
} 
Table 3: Priority profile

\begin{tabular}{|c|c|c|}
\hline$\succeq_{a}^{1}=\succeq_{b}^{1}$ & & $\succeq_{c}^{2}=\succeq_{d}^{2}$ \\
\hline 1 & & 2 \\
2 & & 1 \\
\hline
\end{tabular}

\subsection{Market-wise deferred acceptance (DA) rule}

The deferred acceptance (DA) rule for a single-type market is introduced by Abdulkadiroğlu and Sönmez (2003) who apply Gale and Shapley's (1962) agent-proposing deferred acceptance algorithm in a college admissions problem to an indivisible goods resource allocation problem. Given a type- $t$ profile $R^{t} \in \mathcal{P}\left(X^{t}\right)^{N^{t}}$, the deferred acceptance (DA) type-rule induced by a priority profile $\succeq^{t}$, denoted by $D A^{\succeq^{t}}$, selects a type- $t$ allocation as follows.

Step 1: Each agent applies to the most favorite object according to her preference. Each object selects agents from its applicants up to its quota according to its priority and tentatively keeps them. Any remaining agents are rejected.

Step $k(\geq 2)$ : Each agent who was rejected in the previous step applies to her next favorite object according to her preference. Each object selects agents from its new applicants and the tentatively kept agents up to its quota according to its priority and tentatively keeps them. Any remaining agents are rejected.

The algorithm terminates when no agent is rejected.

Remark 6. For each type $t \in T$ and each type-priority profile $\succeq^{t} \in \mathcal{P}\left(N^{t}\right)^{X^{t}}$, the DA type-rule induced by $\succeq^{t}$ is strategy-proof and non-wasteful (Balinski and Sönmez, 1999), and thus is individually rational by Remark $2 .{ }^{21}$

Definition 3. The market-wise deferred acceptance (DA) rule induced by a priority profile $\succeq=\left(\succeq^{t}\right)_{t \in \boldsymbol{T}}$, denoted by $D A^{\succeq}$, selects its type-t allocation as $D A^{\succeq^{t}}\left(R^{t}\right)$ that is chosen by the DA type-rule induced by type- $t$ priority profile $\succeq^{t}$.

Remark 7. By Remarks 3 and 6, a market-wise DA rule is strategy-proof and individually rational. However, the example in Introduction can be used to show that for some priority profile such as in Table 3, each DA type-rule is Pareto efficient but its market-wise DA is not Pareto efficient.

\subsection{Market-wise serial dictatorship (SD) rule}

The serial dictatorship (SD) rule for a type-t market with respect to a priority order $\geq^{t} \in \mathcal{P}\left(N^{t}\right)$, written as $S D^{\geq^{t}}$, is described as follows: for each type-t preference profile, letting the highestpriority agent with respect to $\geq^{t}$ have her best object, the second-highest-priority agent with respect to $\geq^{t}$ have her best among those remaining, and so on.

\footnotetext{
${ }^{21}$ Ergin (2002) characterizes the priority profiles under which the type- $t$ DA rule is Pareto efficient. Kesten (2006) characterizes the priority profiles under which the type- $t$ TTC rule is fair in terms of envies at the selected type- $t$ allocation.
} 
Note that given a priority order $\geq^{t} \in \mathcal{P}\left(N^{t}\right)$, the serial dictatorship rule for a type- $t$ market with respect to $\geq^{t}$ is strategy-proof and Pareto efficient, and moreover coincides with the TTC and DA rules for a type- $t$ market with the priority profile $\succeq^{t} \in \mathcal{P}\left(N^{t}\right)^{X^{t}}$ in which for each object $x^{t} \in X^{t}, \succeq_{x^{t}}^{t}=\geq^{t}$.

\section{Definition 4. The market-wise serial dictatorship (SD) rule induced by a list of priority}

orders $\geq=\left(\geq^{t}\right)_{t \in \boldsymbol{T}} \in \prod_{t \in \boldsymbol{T}} \mathcal{P}\left(\boldsymbol{N}^{t}\right)$, denoted by $S D^{\geq}$, selects its type- $t$ allocation as $S D^{\geq^{t}}\left(R^{t}\right)$ that is chosen by the SD type-rule induced by type- $t$ priority order $\geq^{t}$.

Remark 8. Note that by Remarks 2 and 3, a market-wise SD rule is strategy-proof and individually rational. However, the example in Introduction can be used to show that it is not Pareto efficient for the priority profile in Table 3.

Remark 9. The serial dictatorship (SD) rule induced by a priority $\geq^{*} \in \mathcal{P}(N)$ selects an allocation for each profile $R \in \mathcal{D}$ as follows: The highest-priority agent under $\geq^{*}$ receives her best bundle, the second-highest-priority agent under $\geq^{*}$ receives her best bundle among remaining objects, and so on.

Note that the SD rule induced by $\geq^{*}$ coincides with the market-wise SD rule induced by $\left(\geq^{t}\right)_{t \in T}$ when the type- $t$ priority $\geq^{t}$ is the same as in $\geq\left.^{*}\right|_{N^{t}}$. In this sense the SD rule is a special case of the market-wise SD rule.

\section{Main Results}

It is known that strategy-proofness and Pareto efficiency are compatible in our setting (Monte and Tumennasan, 2013). The leading example is a serial dictatorship rule in which agents choose their favorite bundle one by one according to a fixed priority order (Remark 9). However, the rule is extremely unfair. For example, when agents have homogeneous preferences and each type object is of unit quotas, the highest priority agent receives her best object for all types which are envied by all of the other agents. A natural way to mitigate this extreme unfairnass is to alternate the dictator across types. As we saw in the previous section, we have a rich class of strategy-proof rules each of whose type rules satisfy a weaker efficiency notion of non-wastefulness. Because these might not be Pareto efficient in general, we turn to a weaker notion of efficiency - second-best efficiency - rather than Pareto efficiency, while focusing on strategy-proof rules: a rule $\varphi$ is second-best incentive compatible if $\varphi$ is strategy-proof and no strategy-proof rule dominates $\varphi$. In other words, a second-best incentive compatible rule is in the Pareto frontier of the set of strategy-proof rules. $^{22}$

We now state the main result of this paper. The proof is in Appendix B.

Theorem 1. Suppose that a rule $\varphi$ is independent and for each $t \in T$, its type rule $\Phi^{t}: \mathcal{P}\left(X^{t}\right)^{N^{t}} \rightarrow$ $\mathcal{A}^{t}$ is strategy-proof and non-wasteful. Then, $\varphi$ is second-best incentive compatible.

\footnotetext{
${ }^{22}$ Our research direction here can be summarized as follows: Find the best (the most efficient) strategy-proof rules. Another natural direction is : Find the best (the most invulnerable to the strategic behaviors) efficient rules. Pathak and Sönmez (2013) and Chen, Egesdal, Pycia, and Yenmez (2014) study how to make a ranking of rules by manipulability among stable rules in a single-type market.
} 
In other words, if we adopt a strategy-proof and non-wasteful rule for each type market, the overall rule is second-best incentive compatible. In this sense, Theorem 1 supports both the independent operation of type markets currently done in most real-life markets, and our current practices in Market Design - independent consideration of the design of each type market.

Since TTC type-rules and DA type-rules are strategy-proof and non-wasteful (Remarks 4 and $6)$, we have the following immediate corollaries.

Corollary 1. For each priority profile $\succeq \in \prod_{t \in T} \mathcal{P}\left(N^{t}\right)^{X^{t}}$, the market-wise top trading cycles rule $T T C^{\succeq}$ is second-best incentive compatible. ${ }^{23}$

Corollary 2. For each priority profile $\succeq \in \prod_{t \in T} \mathcal{P}\left(N^{t}\right)^{X^{t}}$, the market-wise deferred acceptance rule $D A^{\succeq}$ is second-best incentive compatible.

To state our next corollary, we introduce a multiple-type housing market which is a variant of multiple-type markets. ${ }^{24}$ A multiple-type housing market is a multiple-type market $\left(N, T,\left(T_{i}\right)_{i \in N},\left(X^{t}\right)_{t \in T}, q, \omega\right)$ in which the outside options do not necessarily exist. In this paragraph only, we do not assume Assumption 1. Instead we only assume that for each $t \in T$, $\sum_{x^{t} \in X^{t}} q\left(x^{t}\right) \geq\left|N^{t}\right|$, i.e., there are enough objects for each agent to receive an object in each market in which she is interested. The allocation $\omega \in \mathcal{A}$ describes the system of property rights in the economy. We interpret that agent $i$ has the property right for the object $w_{i}^{t}$. Note that the pure distributional case, i.e., no agent has the property right in each market, is a special case of our model. In this case, for each $t \in T$ and each $i \in N^{t}, \omega_{i}^{t}=o^{t}$. Our model is a generalization of the model in Konishi, Quint, and Wako (2001) and Klaus (2008) in the following four points. (i) Some agents may be interested only in a fraction of $T$. (ii) For each $t \in T,\left|X^{t}\right|$ may not be equal to $\left|N^{t}\right|$. (iii) We do not exclude multiple quota. (iv) We do not exclude indifference in preferences. A market-wise top trading cycles rule for a multiple-type housing market is the one induced by a priority profile $\succeq=\left(\succeq^{t}\right)_{t \in T} \in \prod_{t \in T} \mathcal{P}\left(N^{t}\right)^{X^{t}}$ such that for each type $t \in T$ and each type- $t$ object $x^{t}$, if an agent $i \in N^{t}$ is an owner of $x^{t}$, namely $x^{t}=\omega_{i}^{t}$, then for each $j \in N^{t}$ with $j \succeq_{x^{t}}^{t} i, j$ is also an owner of $x^{t}$. In this setup, we have the following result.

Corollary 3. (Klaus, 2008) Every market-wise top trading cycles rule for a multiple-type housing market is second-best incentive compatible. ${ }^{25}$

Our Corollary 3 is more general than Klaus's (2008) original result due to the above four differences in the setup. Furthermore, Theorem 1 is a substantial extension of Klaus's result, as we use non-wastefulness for type rules instead of Pareto efficiency implied by the TTC type-rule. ${ }^{26}$

If we turn to single-type markets, we have the following corollaries:

\footnotetext{
${ }^{23}$ In a single-type market, a TTC rule is in a subclass of Pápai's (2000) hierarchical exchange rules or Pycia and Ünver's (2009) trading cycles rules that are group strategy-proof and Pareto efficient. Thus, by Theorem 1, if we adopt theirs as type rules, the resulting rule is second-best incentive compatible.

${ }^{24}$ This model is sometimes referred to as the generalized Shapley-Scarf housing market. Here, we present a further generalized version of the model in which multiple quotas are allowed.

${ }^{25}$ Rigorously speaking, Corollary 3 is not a direct consequence of Theorem 1 due to Assumption 1 . However, our proof immediately implies Corollary 3, which is given in the Appendix.

${ }^{26}$ To employ non-wastefulness for type rules instead of Pareto efficiency causes a technical difficulty. A discussion on this point can be found in the Appendix.
} 
Corollary 4. (Kesten, 2010) In a single-type market, there is no strategy-proof and Pareto efficient rule that dominates the deferred acceptance rule. ${ }^{27}$

Corollary 5. (Abdulkadiroğlu, Pathak, and Roth, 2009; Kesten and Kurino, 2013; Erdil, 2014). In a single-type market, the deferred acceptance rule is second-best incentive compatible.

Corollary 6. (Erdil, 2014) In a single-type market, a strategy-proof and non-wasteful rule is second-best incentive compatible.

Corollaries 5 and 6 are respectively counterparts of Corollary 2 and Theorem 1 for a singletype market. However, note that we cannot show Corollary 2 (Theorem 1) by applying Corollary 5 (Corollary 6) to each type market. To see this, let us recall the proof technique in Abdulkadiroğlu, Pathak, and Roth (2009) and Erdil (2014): Like we do, they suppose for a contradiction that a strategy-proof rule, $\zeta$, dominates a strategy-proof and non-wasteful rule, $\varphi$. Then they find some agent $i$ and some profile $R$ with $\zeta_{i}(R) P_{i} \varphi_{i}(R) P_{i} o$, and then use a special kind of manipulation to upgrade the outside option between $\zeta_{i}(R)$ and $\varphi_{i}(R)$ in $i$ 's preference, which eventually leads to a contradiction. However, this technique does not work in a multiple-type market as the situation is drastically changed in the following sense: A dominating strategy-proof rule $\zeta$ may assign an object which is worse than the outside option in some type markets, because the domination only requires that the bundle assigned by $\zeta$ is at least as good as the bundle assigned by $\varphi$. That is, it is possible that $\varphi_{i}^{t}(R) P_{i}^{t} o^{t} P_{i}^{t} \zeta_{i}^{t}(R)$ for some type-t. ${ }^{28}$ Note that this situation does not violate $\zeta_{i}(R) R_{i} \varphi_{i}(R)$ in a multiple-type market. Hence each type-rule in a multiple-type market behaves very differently from a rule in a single-type market.

Before we close this section, let us emphasize the technical advantages of our result. Note that our result is valid in various preference domains including the additively separable weak preference domain. It is known that several technical difficulties arise from the indifference in single-type markets (Erdil and Ergin, 2007). Although type preferences are assumed to be strict, Theorem 1 indicates that the second-best incentive compatibility is robust for the indifference with respect to bundles. Furthermore, as pointed out in Kesten and Kurino (2013), the second-best incentive compatibility is sensitive to domain restriction. Since the set of additively separable strict preferences is smaller than the set of all separable strict preferences (See Remark 1), Theorem 1 - more precisely, the preference construction given in Lemma 1 in the Appendix - clarifies a technical limitation of domain restriction for the second-best incentive compatibility result.

\section{$5 \quad$ Market Design Applications}

We briefly describes the representative type-markets and those propsed type-rules discussed in the literature. The type-rules taken up in each subsection are strategy-proof and at least non-wasteful.

\footnotetext{
${ }^{27}$ We note however that Kesten's (2010) original theorem is also valid when the outside option does not exist.

${ }^{28}$ Remember that we assume that $\varphi$ is independent and each type-rule $\Phi^{t}$ is strategy-proof and non-wasteful in Theorem 1. Thus, for each $t \in T, \varphi_{i}^{t}(R) R_{i} o^{t}$.
} 
Theorem 1 implies that an independent rule of using those type-rules in these type markets is second-best incentive compatible.

\subsection{House allocation with existing tenants}

Abdulkadiroğlu and Sönmez (1999) introduce the problem that deals with the on-campus housing assignments for U.S. college students. The problem can be applied not only to the on-campus housing assignment but also the public housing assignment. In this setting, the set $N^{t}$ of agents is divided into the set $N_{N}^{t}$ of newcomers and the set $N_{E}^{t}$ of existing tenants, while objects refer to houses. Corresponding to each existing tenant $i \in N_{E}^{t}$ is a unique occupied house $\omega_{i}^{t} \in X^{t} \backslash\left\{o^{t}\right\}$ which is interpreted as the house that agent $i$ currently lives in. Each newcomer is assumed to occupy the outside option $o^{t}$.

To remedy the welfare losses observed in practice, inspired by Gale's celebrated assignment method, Abdulkadiroğlu and Sönmez (1999) propose the top trading cycles (AS-TTC) typerule induced by a priority ordering $\geq^{t} \in \mathcal{P}\left(N^{t}\right)$. The type-rule is the TTC-type rule induced by the following type- $t$ priority profile $\succeq^{t}$ that is introduced in Section 3.1: ${ }^{29}$ For each $x^{t} \in X^{t}$, let $N^{t}\left(x^{t}\right):=\left\{i \in N^{t} \mid \omega_{i}^{t}=x^{t}\right\}$ be the set of agents who occupy $x^{t}$. Note that $N^{t}\left(x^{t}\right)$ can be empty. Then, for each $x^{t} \in X^{t}, \succeq_{x^{t}}^{t}$ satisfies: (i) $\left.\succeq_{x^{t}}^{t}\right|_{N^{t} \backslash N^{t}\left(x^{t}\right)}=\geq\left.^{t}\right|_{N^{t} \backslash N^{t}\left(x^{t}\right)}$, and (ii) for each $\{i, j\} \subseteq N^{t}$, if $i \in N^{t}\left(x^{t}\right)$ and $j \succeq_{x^{t}}^{t} i$, then $j \in N^{t}\left(x^{t}\right)$.

Abdulkadiroğlu and Sönmez (1999) show that the AS-TTC type-rule is strategy-proof and Pareto efficient, which also follows from Remark 4.

\subsection{Kidney exchange}

Transplantation is the preferred treatment for patients diagnosed with end-stage kidney disease. Roth, Sönmez, and Ünver (2004) introduce the problem that aims to efficiently organize direct exchanges among medically incompatible donor-patient pairs as well as indirect exchanges that involve one incompatible donor-patient and deceased donors. In the setting, agents refer to patients and objects refer to kidneys. Each patient $i \in N^{t}$ is paired with a kidney $k_{i} \in X^{t} \backslash\left\{o^{t}\right\}$ with $q\left(k_{i}\right)=1$ supplied by her intended donor. Thus $\left|N^{t}\right|=\left|X^{t} \backslash\left\{o^{t}\right\}\right|$. Every patient also has the option to enter the waiting list for cadaveric kidneys with priority. Let $w$ be the outside option of waiting lists. ${ }^{30}$

Roth, Sönmez, and Ünver (2004) propose an inventory of top trading cycles and chains (TTCC) type-rules as a plausible generalization of the top trading cycles method to this setting. 31 A cycle is an ordered list $\left(k_{1}, i_{1}, k_{2}, i_{2}, \ldots, k_{m}, i_{m}\right)$ such that kidney $k_{1}$ points to patient $i_{1}$, patient $i_{1}$ points to kidney $k_{2}, \ldots$, kidney $k_{m}$ points to patient $i_{m}$, and patient $i_{m}$ points to kidney

\footnotetext{
${ }^{29}$ We modify the original description of AS-TTC type rule to use our description of the TTC type-rule in Section 3.1.

${ }^{30}$ Unlike the original framework of Roth, Sönmez, and Ünver (2004) that allows for strict preferences, later models of the kidney exchange deal with dichotomous preferences (e.g., Roth, Sönmez, and Ünver, 2005; Yilmaz, 2011).

${ }^{31}$ In what follows, for breavity we do not provide a self-contained and thorough description of TTCC type-rules. We refer the reader to Roth, Sönmez, and Ünver (2004) for a comprehensive account of the rule. See also Sönmez and Ünver (2013).
} 
$k_{1}$. A $\boldsymbol{w}$-chain is an ordered list $\left(k_{1}, i_{1}, k_{2}, i_{2}, \ldots, k_{m}, i_{m}\right)$ such that kidney $k_{1}$ points to patient $i_{1}$, patient $i_{1}$ points to kidney $k_{2}, \ldots$, kidney $k_{m}$ points to patient $i_{m}$, and patient $i_{m}$ points to $w$.

The TTCC algorithm is based on iteratively identifying cycles and $w$-chains in a directed graph and carrying out the induced trades. ${ }^{32}$ The way $w$-chains are handled in the algorithm depends on the so-called chain selection rule. Roth, Sönmez, and Ünver (2004) discuss various chain selection rules and investigate their implications for welfare and incentives. Of particular interest to us among these are those rules that induce strategy-proofness and Pareto efficiency of TTCC.

\subsection{School choice}

Abdulkadiroğlu and Sönmez (2003) introduce the problem that concerns student assignment to public schools. In this context agents refer to the students, objects refer to the schools, and the outside option represents other education options such as private schools. Additionally, each school $x^{t} \in X^{t}$ has a priority $\succeq_{x^{t}}^{t}$ over students which is a linear order in $\mathcal{P}\left(N^{t}\right)$.

A school choice problem (Abdulkadiroğlu and Sönmez, 2003) is a type market with a typepriority profile. Thus our descriptions of the TTC type-rule and the DA type-rule in Section 3 are for a school choice problem. Thus the TTC type-rule is strategy-proof and Pareto efficient (Remark 4) and the DA type-rule is strategy-proof and non-wasteful (Remark 6).

\section{Conclusion}

In this paper, we consider efficiency for strategy-proof rules in a multiple-type market. The full efficiency is strong enough that we end up with an extremely unfair rule such as the serial dictatorship (Monte and Tumennasan, 2013). We turn to a weaker efficiency notion of the second-best incentive compatibility that requires a rule to be strategy-proof and not be dominated by any other strategy-proof rules. Our main result is the second-best incentive compatibility of a market-wise application of non-wasteful and strategy-proof type rules that include the two well-known prioritybased rules of top trading cycles (TTC) and deferred acceptance (DA). This result shows a rich class of simple and reasonable strategy-proof rules are included in the Pareto frontier of strategyproof rules, and moreover supports our practices of designing a type rule to be strategy-proof and at least non-wasteful in Matching Market Design.

We now discuss the existence of the outside options in Assumption 1. As Kesten and Kurino (2013) point out, Corollary 6 does not hold when there is no outside option: For example, consider a single-type market with $n$ agents and $n$ objects with unit quotas. Then, a constant allocation rule is strategy-proof and non-wasteful. However, the rule is dominated by the corresponding core rule which is also strategy-proof. With the same logic, our main result of Theorem 1 no longer holds

\footnotetext{
${ }^{32}$ Although there are clear similarities between the AS-TTC type-rule in a house allocation problem with existing tenants and the TTCC type-rule in the kidney exchange problem, the adaptations of the top trading cycles method differ in terms of the role the outside option plays. In the former context the outside option always points to the highest priority agent, whereas in the latter context, the $w$-option never points to any agent. This subtle nuance is one main source of the difference between the two rules, and the reason why TTCC type-rules are not described as a special case of the TTC type-rules introduced in Section 3.1.
} 
without Assumption 1. As Kesten and Kurino (2013) show, for a single-type market without the outside option, the DA rule is second-best incentive compatible. It is an interesting open question to prove that for a multiple-type market without outside options, the market-wise DA rule is second-best incentive compatible.

In this paper we have focused on deterministic rules. There is a growing literature on lottery rules in matching problems (e.g., Abdulkadiroğlu and Sönmez, 1998; Bogomolnaia and Moulin, 2001; Che and Kojima, 2010). Although the counterpart of Theorem 1 for lottery rules for a single-type market are still true, Erdil (2014) show that the counterpart cannot be applied to an interesting class of lottery rules such as the random serial dictatorship (RSD) which randomly selects a priority and implements the serial dictatorship for the realized priority because RSD is wasteful. Moreover, we have not yet known any strategy-proof and non-wasteful lottery rule even in a single-type market. Hence we doubt the usefulness of the extension of our Theorem 1 to lottery rules in a multiple-type market. Therefore the future direction that we could take in the same spirit of our paper is to find a general strategy-proof rule dominating a practical strategy-proof rule. For a single-type market, Erdil constructed an artificial lottery rule that is strategy-proof and dominates RSD. However, his rule is quite limited to a small economy and a general dominating rule against stochastic DA or TTC is not known yet. Thus it is an interesting open question as to how much room we can have against those interesting lottery rules for efficiency while keeping strategy-proofness. We believe that this paper could be a benchmark in this direction, and could clarify technical limitations and provide technical tools for the question.

\section{A Appendix: Proof of Remark 1}

Claim 1. $\mathcal{P}_{\text {add }}\left(X_{i}\right) \neq \mathcal{P}_{\text {sep }}\left(X_{i}\right)$.

Proof. The proof idea is similar to the one in page 43 in Fishburn (1970) that shows $\mathcal{R}_{\text {add }}\left(X_{i}\right) \subsetneq$ $\mathcal{R}_{\text {sep }}\left(X_{i}\right)$. Suppose that $X^{1}=X^{2}=\{x, y, z\}$ and $X_{i}=X^{1} \times X^{2}$. Let $R_{i}$ be the preference such that

$$
(x, x) P_{i}(x, y) P_{i}(y, x) P_{i}(z, x) P_{i}(y, y) P_{i}(x, z) P_{i}(y, z) P_{i}(z, y) P_{i}(z, z) .
$$

Obviously $R_{i} \in \mathcal{P}_{\text {sep }}\left(X_{i}\right)$. We show $R_{i} \notin \mathcal{P}_{\text {add }}\left(X_{i}\right)$. Suppose to the contrary that $\left(u^{1}, u^{2}\right) \in \mathcal{U}^{1} \times \mathcal{U}^{2}$ represents $R_{i}$. Since $(z, x) P_{i}(x, z)$ and $(y, z) P_{i}(z, y), u^{1}(z)+u^{2}(x)>u^{1}(x)+u^{2}(z)$ and $u^{1}(y)+u^{2}(z)>u^{1}(z)+u^{2}(y)$. Thus $u^{1}(z)+u^{2}(x)+u^{1}(y)+u^{2}(z)>u^{1}(x)+u^{2}(z)+u^{1}(z)+u^{2}(y)$. Cancelling out $u^{1}(z)$ and $u^{2}(z)$, we obtain $u^{1}(y)+u^{2}(x)>u^{1}(x)+u^{2}(y)$. This violates that $(x, y) P_{i}(y, x)$.

\section{B Appendix: Proof of Theorem 1}

We first introduce some notations: For each $\succsim \in \mathcal{R}(Y)$ and each $y \in Y$, let $\mathrm{UC}(\succsim, y), \operatorname{SUC}(\succsim, y)$, $\mathrm{LC}(\succsim, y)$ and $\operatorname{SLC}(\succsim, y)$ be the upper, strict upper, lower, and strict lower contour set of $\succsim$ at $y$, 
respectively. That is, $\mathrm{UC}(\succsim, y):=\{z \in Y \mid z \succsim y\}, \operatorname{SUC}(\succsim, y):=\{z \in Y \mid z \succsim y$ and not $y \succsim z\}$, $\mathrm{LC}(\succsim, y):=\{z \in Y \mid y \succsim z\}$ and $\operatorname{SLC}(\succsim, y):=\{z \in Y \mid y \succsim z$ and not $z \succsim y\}$.

Before we prove Theorem 1, we provide four lemmas. Lemma 1 states that the domain $\mathcal{D}$ is rich enough to choose the preferences we need in the proofs of subsequent lemmas and theorem.

Lemma 1. Let $i \in N$. Let $\left(\tilde{R}_{i}^{t}\right) \in \prod_{t \in T_{i}} \mathcal{P}\left(X^{t}\right)$ and $\tilde{x}_{i} \in X_{i}$. There exists $R_{i} \in \mathcal{P}_{\text {add }}\left(X_{i}\right)$ such that

(i) $\forall t \in T_{i}, R_{i}^{t}=\tilde{R}_{i}^{t}$ and

(ii) $\forall x_{i} \in X_{i},\left[\left\{\exists t \in T_{i}\right.\right.$ s.t. $\left.\left.x_{i}^{t} \in S L C\left(\tilde{R}_{i}^{t}, \tilde{x}_{i}^{t}\right)\right\} \Rightarrow \tilde{x}_{i} P_{i} x_{i}\right]$.

Proof. Without loss of generality, suppose that $T_{i}=T$. For each $t \in T$, let $X^{t}=\left\{x^{t 1}, \cdots, x^{t\left|X^{t}\right|}\right\}$, and assume, without loss of generality, that $x^{t 1} \tilde{R}_{i}^{t} x^{t 2} \tilde{R}_{i}^{t} \cdots \tilde{R}_{i}^{t} x^{t \mid X^{t}}$. For each $t \in T$, let $k_{t} \in \mathbb{Z}_{++}$be the cardinality of $\operatorname{UC}\left(\tilde{R}_{i}^{t}, \tilde{x}_{i}^{t}\right)$ where $x^{t k_{t}}=\tilde{x}_{i}^{t}$. Let $k_{t}^{\prime}:=\left|X^{t}\right|-k_{t}, K:=\sum_{t \in T} k_{t}$ and $K^{\prime}:=\sum_{t \in T} k_{t}^{\prime}$. Define $\left(u_{i}^{t}\right) \in \prod_{t \in T} \mathcal{U}^{t}$ as follows:

$$
\begin{array}{cccc}
u_{i}^{1}\left(x^{11}\right)=10^{K-1}, & u_{i}^{1}\left(x^{12}\right)=10^{K-2}, & \cdots & u_{i}^{1}\left(x^{1 k_{1}}\right)=10^{K-k_{1}}, \\
u_{i}^{2}\left(x^{21}\right)=10^{K-\left(k_{1}+1\right)}, & u_{i}^{2}\left(x^{22}\right)=10^{K-\left(k_{1}+2\right)}, & \cdots & u_{i}^{2}\left(x^{2 k_{2}}\right)=10^{K-\left(k_{1}+k_{2}\right),}, \\
\vdots & \vdots & & \vdots \\
u_{i}^{|T|}\left(x^{|T| 1}\right)=10^{K-\left(\sum_{t=1}^{|T|-1} k_{t}+1\right)}, & u_{i}^{|T|}\left(x^{|T| 2}\right)=10^{K-\left(\sum_{t=1}^{|T|-1} k_{t}+2\right)}, & \cdots & u_{i}^{|T|}\left(x^{|T| k_{|T|}}\right)=10^{K-\left(\sum_{t \in T} k_{t}\right)}\left(=10^{0}\right) .
\end{array}
$$

Let $C^{\prime}:=2 \sum_{t \in T} u_{i}^{t}\left(x^{t 1}\right)$ and $C:=C^{\prime}+1 .{ }^{33}$ In the following, for each $t \in T$, if $k_{t}^{\prime}=0$, then the corresponding row should be skipped.

$$
\begin{aligned}
& u_{i}^{1}\left(x^{1\left(k_{1}+1\right)}\right)=\frac{1}{10}-C, \\
& u_{i}^{2}\left(x^{2\left(k_{2}+1\right)}\right)=\frac{1}{10^{k_{1}^{\prime}+1}}-C, \\
& u_{i}^{1}\left(x^{1\left(k_{1}+2\right)}\right)=\frac{1}{10^{2}}-C, \\
& u_{i}^{2}\left(x^{2\left(k_{2}+2\right)}\right)=\frac{1}{10^{k_{1}^{\prime}+2}}-C, \\
& \cdots \quad u_{i}^{1}\left(x^{1\left|X^{1}\right|}\right)=\frac{1}{10^{k_{1}^{\prime}}}-C, \\
& \vdots \\
& u_{i}^{|T|}\left(x^{|T|\left(k_{|T|}+1\right)}\right)=\frac{1}{10^{\sum_{t=1}^{\mid T-1} k_{t}^{\prime}+1}}-C, \\
& u_{i}^{|T|}\left(x^{|T|\left(k_{|T|}^{\prime}+1\right)}\right)=\frac{1}{10^{\sum_{t=1}^{|T|-1} k_{t}^{\prime}+2}}-C, \\
& u_{i}^{2}\left(x^{2\left|X^{2}\right|}\right)=\frac{1}{10^{k_{1}^{\prime}+k_{2}^{\prime}}}-C \text {, }
\end{aligned}
$$

Now, we define $R_{i} \in \mathcal{R}\left(X_{i}\right)$ as follows: for each $\left\{y_{i}, z_{i}\right\} \subseteq X_{i}$,

$$
y_{i} R_{i} z_{i} \Leftrightarrow \sum_{t \in T} u_{i}^{t}\left(y_{i}^{t}\right) \geq \sum_{t \in T} u_{i}^{t}\left(z_{i}^{t}\right)
$$

Obviously, $R_{i} \in \mathcal{R}_{a d d}\left(X_{i}\right) \subseteq \mathcal{R}_{\text {sep }}\left(X_{i}\right)$. It is also obvious that $\left(R_{i}^{t}\right)_{t \in T}=\left(\tilde{R}_{i}^{t}\right)_{t \in T}$, i.e., Item (i) is satisfied.

We show that $R_{i}$ satisfies Item (ii). Let $y_{i} \in X_{i}$ be such that for some $t \in T, y_{i}^{t} \in \operatorname{SLC}\left(R_{i}^{t}, \tilde{x}_{i}^{t}\right)$. Note that $y_{i}$ has at least one coordinate whose utility contains the $-C$ term while $\tilde{x}_{i}$ does not. Therefore, $\sum_{t \in T} u_{i}^{t}\left(\tilde{x}_{i}^{t}\right)>0>\sum_{t \in T} u_{i}^{t}\left(y_{i}^{t}\right)$. Thus, $\tilde{x}_{i} P_{i} y_{i}$. Thus, Item (ii) is satisfied.

Finally, we prove that $R_{i} \in \mathcal{P}_{\text {add }}\left(X_{i}\right)$. By construction, $R_{i} \in \mathcal{R}_{\text {add }}\left(X_{i}\right)$. Since $\mathcal{P}_{\text {add }}\left(X_{i}\right)=$ $\mathcal{R}_{a d d}\left(X_{i}\right) \cap \mathcal{P}\left(X_{i}\right)$, we need to show $R_{i} \in \mathcal{P}\left(X_{i}\right)$. Let $y_{i}, z_{i}$ be such that $\sum_{t \in T} u_{i}^{t}\left(y_{i}^{t}\right)=\sum_{t \in T} u_{i}^{t}\left(z_{i}^{t}\right)$.

\footnotetext{
${ }^{33}$ Note that $C^{\prime}$ is greater than the utility obtained by the bundle $\left(x^{11}, \cdots, x^{|T| 1}\right)$ which is the best bundle according to the resulting preference $R_{i}$.
} 
First, we claim that the number of types in which the type object is worse than $\tilde{x}_{i}^{t}$ is the same between $y_{i}$ and $z_{i}$.

Claim 2. $\left|\left\{t \in T \mid \tilde{x}_{i}^{t} P_{i}^{t} y_{i}^{t}\right\}\right|=\left|\left\{t \in T \mid \tilde{x}_{i}^{t} P_{i}^{t} z_{i}^{t}\right\}\right|$.

Let $\alpha:=\left|\left\{t \in T \mid \tilde{x}_{i}^{t} P_{i}^{t} y_{i}^{t}\right\}\right|$ and $\beta:=\left|\left\{t \in T \mid \tilde{x}_{i}^{t} P_{i}^{t} z_{i}^{t}\right\}\right|$. Suppose to the contrary that $\alpha \neq \beta$. Assume, without loss of generality, that $\alpha<\beta$. We can decompose the utility into three parts:

$$
\sum_{t \in T} u_{i}^{t}\left(y_{i}^{t}\right)=\sum_{\substack{t \in T \\ t \in T \\ y_{i}^{t} R_{i}^{t} \tilde{x}_{i}^{t}}} u_{i}^{t}\left(y_{i}^{t}\right)+\left(\sum_{\substack{t \in T \\ \tilde{x}_{i}^{t} P_{i}^{t} y_{i}^{t}}} u_{i}^{t}\left(y_{i}^{t}\right)+\alpha C\right)-\alpha C
$$

Since $0 \leq\left(\sum_{\tilde{x}_{i}^{t} P_{i}^{t} y_{i}^{t}} u_{i}^{t}\left(y_{i}^{t}\right)+\alpha C\right)<1$, we have

$$
\sum_{\substack{t \in T \\ t \in T \\ y_{i}^{t} R_{i}^{t} \tilde{x}_{i}^{t}}} u_{i}^{t}\left(y_{i}^{t}\right)-\alpha C \leq \sum_{t \in T} u_{i}^{t}\left(y_{i}^{t}\right) \leq \sum_{\substack{t \in T \\ t \in T \\ y_{i}^{t} R_{i}^{t} \tilde{x}_{i}^{t}}} u_{i}^{t}\left(y_{i}^{t}\right)+1-\alpha C
$$

Similarly, we have

$$
\sum_{\substack{t \in T \\ z_{i}^{t} R_{i}^{t} \tilde{x}_{i}^{t}}} u_{i}^{t}\left(z_{i}^{t}\right)-\beta C \leq \sum_{t \in T} u_{i}^{t}\left(z_{i}^{t}\right) \leq \sum_{\substack{t \in T \\ z_{i}^{t} R_{i}^{t} \tilde{x}_{i}^{t}}} u_{i}^{t}\left(z_{i}^{t}\right)+1-\beta C .
$$

Thus, we have

$$
\begin{aligned}
& \left(\sum_{\substack{t \in T \\
y_{i}^{t} R_{i}^{t} \tilde{x}_{i}^{t}}} u_{i}^{t}\left(y_{i}^{t}\right)-\alpha C\right)-\left(\sum_{\substack{t \in T \\
z_{i}^{t} R_{i}^{t} \tilde{x}_{i}^{t}}} u_{i}^{t}\left(z_{i}^{t}\right)+1-\beta C\right)=-1+\left(\sum_{\substack{t \in T \\
y_{i}^{t} R_{i}^{x_{i}^{t}}}} u_{i}^{t}\left(y_{i}^{t}\right)-\sum_{\substack{t \in T \\
z_{i}^{t} R_{i}^{t} \tilde{x}_{i}^{t}}} u_{i}^{t}\left(z_{i}^{t}\right)\right)+(\beta-\alpha) C \\
& \geq-1+\left(-\frac{1}{2} C^{\prime}\right)+(\beta-\alpha) C \quad\left(\because \sum_{\substack{t \in T \\
y_{i}^{t} R_{i}^{t} \tilde{x}_{i}^{t}}} u_{i}^{t}\left(y_{i}^{t}\right)-\sum_{\substack{t \in T \\
z_{i}^{t} R_{i}^{t} \tilde{x}_{i}^{t}}} u_{i}^{t}\left(z_{i}^{t}\right) \geq-\frac{1}{2} C^{\prime}\right) \\
& =-1+\left(-\frac{1}{2} C^{\prime}\right)+(\beta-\alpha) C^{\prime}+(\beta-\alpha) \geq \frac{1}{2} C^{\prime}>0 . \quad(\because \alpha<\beta)
\end{aligned}
$$

Thus, $\sum_{t \in T} u_{i}^{t}\left(z_{i}^{t}\right)<\sum_{t \in T} u_{i}^{t}\left(y_{i}^{t}\right)$, a contradiction. Thus, $\alpha=\beta$. The proof of the Claim is completed.

Now we complete the proof of $R_{i} \in \mathcal{P}\left(X_{i}\right)$. Let us express $Y:=\sum_{t \in T} u_{i}^{t}\left(y_{i}^{t}\right)+\alpha C$ as a $\left(K+K^{\prime}\right)$ digits rational number. That is, $Y=Y_{1} Y_{2} \cdots Y_{K} \cdot Y_{K+1} Y_{K+2} \cdots Y_{K+K^{\prime}} .{ }^{34}$ Note that by the construction, each digit is equal to 1 or 0 . Note also that $\left(Y_{1}, \cdots, Y_{k_{1}}, Y_{K+1}, \cdots, Y_{K+k_{1}^{\prime}}\right)$ tells us which type- 1 object is assigned at $y_{i}$ since for each $k \in\left\{1, \cdots k_{1}, K+1, \cdots, K+k_{1}^{\prime}\right\}, Y_{k}=1$ if and only if

$$
y_{i}^{1}= \begin{cases}x^{1 k} & \text { if } 1 \leq k \leq k_{1} \\ x^{1\left(k-K+k_{1}\right)} & \text { otherwise }\end{cases}
$$

In general, for $t \geq 2,\left(Y_{\sum_{t^{\prime}<t} k_{t^{\prime}}+1}, \cdots, Y_{\sum_{t^{\prime} \geq t} k_{t^{\prime}}}, Y_{K+\sum_{t^{\prime}<t} k_{t^{\prime}}^{\prime}+1}, \cdots, Y_{K+\sum_{t^{\prime} \geq t} k_{t^{\prime}}^{\prime}}\right)$ tells us the type$t$ object at $y_{i}$. Therefore, we can identify the bundle $y_{i}$ with the value of $Y$. Similarly, let

\footnotetext{
${ }^{34}$ Note that $Y_{1}$ denotes the $10^{K-1}$ 's place of $Y$ (which may be 0 ), $Y_{2}$ denotes the $10^{K-2}$ 's place of $Y$ (which may be 0 ) and so on. Similarly, $Y_{K+1}$ denotes the $\frac{1}{10}$ 's place of $Y, Y_{K+2}$ denotes the $\frac{1}{10^{2}}$ 's place of $Y$ and so on.
} 
$Z:=\sum_{t \in T} u_{i}^{t}\left(z_{i}^{t}\right)+\beta C$ and

$$
Z=Z_{1} Z_{2} \cdots Z_{K} \cdot Z_{K+1} Z_{K+2} \cdots Z_{K+K^{\prime}}
$$

Since $\sum_{t \in T} u_{i}^{t}\left(y_{i}^{t}\right)=\sum_{t \in T} u_{i}^{t}\left(z_{i}^{t}\right)$ and $\alpha=\beta\left(\because\right.$ Claim), we have $Y=\sum_{t \in T} u_{i}^{t}\left(y_{i}^{t}\right)+\alpha C=$ $\sum_{t \in T} u_{i}^{t}\left(z_{i}^{t}\right)+\beta C=Z$. Thus, $y_{i}=z_{i}$.

We introduce notations: For each $i \in N$, each $t \in T_{i}$, each $R_{i}^{t} \in \mathcal{P}\left(X^{t}\right)$, each $R_{i} \in \mathcal{D}_{i}$ and each $R \in \mathcal{D}$, let

$$
\begin{aligned}
B\left(R_{i}^{t}\right) & :=\left|\operatorname{SUC}\left(R_{i}^{t}, o^{t}\right)\right|, \\
B\left(R_{i}\right) & :=\sum_{t \in T_{i}} B\left(R_{i}^{t}\right), \text { and } \\
B(R) & :=\sum_{i \in N} B\left(R_{i}\right) .
\end{aligned}
$$

Namely, $B$ is the operator that assigns the number of object(s) which are preferred to the outside option(s). For each $R \in \mathcal{D}$, let $I(R)$ be the number of agents whose preferences are not strict at $R$, i.e.,

$$
I(R):=\left|\left\{i \in N \mid R_{i} \notin \mathcal{P}\left(X_{i}\right)\right\}\right| .
$$

The following two notions are the key to the proof of Theorem 1 . Given $R \in \mathcal{D}$, an allocation $a \in \mathcal{A}$ coordinate-wise weakly dominates $b \in \mathcal{A}$ at $R$, written as $a \mathrm{cw}-\operatorname{dom}(R) b$, if

$$
\forall i \in N, \forall t \in T_{i}, a_{i}^{t} R_{i}^{t} b_{i}^{t}
$$

Given a pair of rules $(\zeta, \varphi)$, a profile $R \in \mathcal{D}$ satisfies the $(\zeta, \varphi)$-reverse property if

$$
\exists i \in N \text { s.t. }\left[\zeta_{i}(R) P_{i} \varphi_{i}(R) \text { and }\left\{\exists t \in T_{i} \text { s.t. } \varphi_{i}^{t}(R) P_{i}^{t} \zeta_{i}^{t}(R)\right\}\right] .{ }^{35}
$$

Let us sketch the proof of Theorem 1. ${ }^{36}$ The proof shall be done by a contradiction. Therefore, we assume that there exists a strategy-proof rule $\zeta$ which dominates $\varphi$. Let $R^{(1)} \in \mathcal{D}$ be such that $\zeta\left(R^{(1)}\right)$ dominates $\varphi\left(R^{(1)}\right)$ at $R^{(1)}$. Starting from this, we inductively construct two sequences of non-negative integers $\left\{N^{(k)}\right\}_{k=0}^{\infty}$ and $\left\{B^{(k)}\right\}_{k=1}^{\infty}$ satisfying Items (seq-i) and (seq-ii).

(seq-i) $\left\{N^{(k)}\right\}_{k=0}^{\infty}$ is weakly decreasing, i.e., $N^{(0)} \geq N^{(1)} \geq N^{(2)} \geq \cdots$.

(seq-ii) If $N^{(k-1)}$ does not decrease $\left(N^{(k-1)}=N^{(k)}\right)$, then the corresponding part of $\left\{B^{(k)}\right\}_{k=1}^{\infty}$ decreases, i.e., for each $k \in \mathbb{N}$, if $N^{(k-1)}=N^{(k)}$, then $B^{(k)}>B^{(k+1)}$. ${ }^{37}$

In each induction step of the proof, we shall choose an agent $i^{(k)}$ and her new preference $R_{i^{(k)}}^{(k+1)}$, and define $N^{(k)}:=\left|N \backslash\left\{i^{(1)}, \cdots, i^{(k)}\right\}\right|$ and $B^{(k+1)}:=B\left(R_{i^{(k)}}^{(k+1)} ; R_{-i(k)}^{(k)}\right)$. It is a process of successive

\footnotetext{
${ }^{35}$ Note that if $\zeta \operatorname{dom} \varphi$ and $R \in \mathcal{D}$ satisfies $(\zeta, \varphi)$-reverse property, then $\zeta(R) \operatorname{dom}(R) \varphi(R)$.

${ }^{36}$ Our proof is greatly inspired by the one in Klaus (2008).

${ }^{37}$ Note that these sequences cause a contradiction since sequences of non-negative integers cannot meet both (seq-i) and (seq-ii).
} 
preference replacements to satisfy Item (seq-ii) as Item (i) is automatically satisfied by definition, which is made possible by the $(\zeta, \varphi)$-reverse property at the profile under consideration. ${ }^{38}$ The simplest case is when every profile satisfies the reverse property. One such simple case is: each type-rule $\Phi^{t}$ is Pareto efficient and $\mathcal{D}=\prod_{i \in N} \mathcal{P}_{\text {sep }}\left(X_{i}\right) .{ }^{39}$

However, the assumptions of Theorem 1 navigate away the above situation in two ways. First, we only assume non-wastefulness for each type rule instead of Pareto efficiency. Second, the preference domain contains weak preferences. Even under one of these weak assumptions, it is easy to show that a given preference profile may not satisfy the $(\zeta, \varphi)$-reverse property. For this reason, in addition to the process constructing $\left\{N^{(k)}\right\}_{k=0}^{\infty}$ and $\left\{B^{(k)}\right\}_{k=1}^{\infty}$ (let us call it the constructing process), we need another process to transit from a profile without the $(\zeta, \varphi)$-reverse property toward a profile with the $(\zeta, \varphi)$-reverse property (let us call it the transition process). Lemmas 2 to 4 show how we transit from one profile to another. Under a given profile without the $(\zeta, \varphi)$-reverse property, we use two types of transition according to whether the coordinatewise weak domination occurs in the profile or not. Lemma 2 describes the transition when the domination occurs, while Lemma 3 describes when the domination does not. Lemma 4 guarantees that repeating these transitions, we finally reach a profile with the $(\zeta, \varphi)$-reverse property. In sum, the proof of Theorem 1 is the constructing process in which each step contains the transition process. 40

Note that both the constructing process and the transition process contain preference replacements of agents. When we prove that the resulting sequences satisfy (seq-ii), we need the following: once an agent is involved in the preference replacement with respect to the constructing process, she is never involved in the preference replacement with respect to the subsequent transition process. In other words, we need to design the transition process so as not to disturb the constructing process. This trick is realized by Items (lem2-1), (lem3-1) and Item (i) in Lemma 4.

Now, we present Lemma 2. It tells us how we transit from a profile if the coordinate-wise weak domination occurs in the profile. Item (lem2-2) is needed to terminate the induction process in Lemma 4 in a finite number of steps, and it is also needed to prove Item (ii) in Lemma 4, which is also a trick to prove that two sequences in the proof of Theorem 1 satisfies (seq-ii). Item (lem2-3) guarantees that the induction argument in Lemma 4 bites.

Lemma 2. Suppose that a rule $\varphi$ is independent and for each $t \in T, \Phi^{t}: \mathcal{P}\left(X^{t}\right)^{N^{t}} \rightarrow \mathcal{A}^{t}$ is strategy-proof and non-wasteful. Suppose also that a strategy-proof rule $\zeta$ dominates $\varphi$. Let $R \in \mathcal{D}$ be such that $\zeta(R) \operatorname{dom}(R) \varphi(R)$. If $\zeta(R)$ cw-dom $(R) \varphi(R)$, then there exist $i \in N$ and $R_{i}^{\prime} \in \mathcal{D}_{i}$ such that

(lem2-1) $\exists t \in T_{i}$ s.t. $\zeta_{i}^{t}(R) P_{i}^{t} \varphi_{i}^{t}(R) P_{i}^{t} o^{t}$,

(lem2-2) $B\left(R_{i}^{\prime} ; R_{-i}\right)<B(R)$ and $I\left(R_{i}^{\prime} ; R_{-i}\right) \leq I(R)$, and

\footnotetext{
${ }^{38}$ The detailed construction of a new preference is indicated by conditions $\left(1^{*}-i\right),\left(1^{*}-i i\right),\left(k^{*}-i\right)$ and $\left(k^{*}-i i\right)$ in the proof of Theorem 1 .

${ }^{39}$ See Lemma 1 in Klaus (2008).

${ }^{40}$ Therefore, the profiles in the proof of Theorem 1 are doubly indexed. The first index indicates the steps of the constructing process, and the second index indicates the steps of the transition process.
} 
(lem2-3) $\zeta\left(R_{i}^{\prime} ; R_{-i}\right) \operatorname{dom}\left(R_{i}^{\prime} ; R_{-i}\right) \varphi\left(R_{i}^{\prime} ; R_{-i}\right)$

Proof. First, we show that there exists $i \in N$ satisfying (lem2-1). Suppose to the contrary that for each $i \in N$ and each $t \in T_{i},\left[\varphi_{i}^{t}(R) P_{i}^{t} o^{t} \Rightarrow \varphi_{i}^{t}(R) R_{i}^{t} \zeta_{i}^{t}(R)\right]$. Since $\zeta(R)$ cw-dom $(R) \varphi(R)$, the hypothesis is equivalent to

$$
\forall i \in N, \forall t \in T_{i},\left[\varphi_{i}^{t}(R) P_{i}^{t} o^{t} \Rightarrow \varphi_{i}^{t}(R)=\zeta_{i}^{t}(R)\right]
$$

Claim 3. $\forall i \in N, \forall t \in T_{i},\left[\varphi_{i}^{t}(R)=o^{t} \Rightarrow \zeta_{i}^{t}(R)=o^{t}\right]$.

Suppose not. Let $i \in N$ and $t \in T_{i}$ be such that $\varphi_{i}^{t}(R)=o^{t}$ and $\zeta_{i}^{t}(R) \neq o^{t}$. Let $x^{t}:=\zeta_{i}^{t}(R)$ and $a^{t}:=\left(\varphi_{i}^{t}(R)\right)_{i \in N^{t}}$. We show that at least one unit of the object $x^{t}$ is not assigned to any agent at the type- $t$ allocation $a^{t}$. By (1), for each $j \in N^{t},\left[a_{j}^{t}=x^{t} \Rightarrow \zeta_{j}^{t}(R)=x^{t}\right]$. Thus, since $a_{i}^{t}=o^{t}$ and $\zeta_{i}^{t}(R)=x^{t}$, we have $\left|\left\{j \in N^{t} \mid a_{j}^{t}=x^{t}\right\}\right|<\left|\left\{j \in N^{t} \mid \zeta_{j}^{t}(R)=x^{t}\right\}\right| \leq q\left(x^{t}\right)$. However, since $\zeta(R) \mathrm{cw}-\operatorname{dom}(R) \varphi(R)$ and type preferences are strict, $x^{t}=\zeta_{i}^{t}(R) P_{i}^{t} \varphi_{i}^{t}(R)=\Phi_{i}^{t}\left(R^{t}\right)$, a violation of non-wastefulness of $\Phi^{t}$. This completes the proof of the Claim.

Note that by Remark 2, $\Phi^{t}$ is individually rational. Thus, by (1) and the Claim, for each $i \in N$ and each $t \in T_{i}, \varphi_{i}^{t}(R)=\zeta_{i}^{t}(R)$. Thus, $\varphi(R)=\zeta(R)$, which violates our assumption that $\zeta(R) \operatorname{dom}(R) \varphi(R)$. Therefore, there exists $i \in N$ satisfying (lem2-1).

Next, let $i \in N$ and $t_{0} \in T_{i}$ be such that $\zeta_{i}^{t_{0}}(R) P_{i}^{t_{0}} \varphi_{i}^{t_{0}}(R) P_{i}^{t_{0}} o^{t_{0}}$. We show that there exists a preference $R_{i}^{\prime} \in \mathcal{D}_{i}$ which satisfies (lem2-2). First, we define a list of type preferences $\left(\tilde{R}_{i}^{\prime t}\right)_{t \in T_{i}}$. In words, $\left(\tilde{R}_{i}^{\prime t}\right)_{t \in T_{i}}$ is obtained from $\left(R_{i}^{t}\right)_{t \in T_{i}}$ by changing the ranking of $o^{t_{0}}$ just above $\varphi_{i}^{t_{0}}(R)$ while the relative rankings of any other objects stay the same. Formally, $\left(\tilde{R}_{i}^{\prime t}\right)_{t \in T_{i}}$ is defined as follows;

- $\left[\forall t \in T_{i} \backslash\left\{t_{0}\right\}, \tilde{R}_{i}^{\prime t}=R_{i}^{t}\right]$ and $\left.\tilde{R}_{i}^{t_{0}}\right|_{\left(X^{t_{0}} \backslash\left\{o^{t_{0}}\right\}\right) \times\left(X^{t_{0}} \backslash\left\{o_{0} t_{0}\right\}\right)}=\left.R_{i}^{t_{0}}\right|_{\left(X^{t_{0}} \backslash\left\{o^{t_{0}}\right\}\right) \times\left(X^{t_{0}} \backslash\left\{o^{t_{0}}\right\}\right)}$,

- $o^{t_{0}} \tilde{P}_{i}^{\prime t_{0}} \varphi_{i}^{t_{0}}(R)$ and

- $\nexists x^{t_{0}} \in X^{t_{0}} \backslash\left\{o^{t_{0}}, \varphi_{i}^{t_{0}}(R)\right\}$ s.t. $o^{t_{0}} \tilde{R}_{i}^{\prime t_{0}} x^{t_{0}} \tilde{R}_{i}^{t_{0}} \varphi_{i}^{t_{0}}(R)$.

By applying Lemma 1 for $\left(\tilde{R}_{i}^{\prime t}\right)_{t \in T_{i}}$ and $\zeta_{i}(R)$, we can choose a preference $R_{i}^{\prime} \in \mathcal{P}_{\text {add }}\left(X_{i}\right)$ such that

$$
\forall x_{i} \in X_{i},\left[\left\{\exists t \in T_{i} \text { s.t. } x_{i}^{t} \in \operatorname{SLC}\left(R_{i}^{\prime t}, \zeta_{i}^{t}(R)\right)\right\} \Rightarrow \zeta_{i}(R) P_{i}^{\prime} x_{i}\right]
$$

By (lem2-1) and the construction of $\left(R_{i}^{\prime t}\right)_{t \in T_{i}}, B\left(R_{i}^{\prime}\right)<B\left(R_{i}\right)$. Therefore, $B\left(R_{i}^{\prime} ; R_{-i}\right)<B(R)$. Since $R_{i}^{\prime} \in \mathcal{P}_{\text {add }}\left(X_{i}\right), I\left(R_{i}^{\prime} ; R_{-i}\right) \leq I(R)$.

Finally, we show (lem2-3). Since $\Phi^{t_{0}}$ is strategy-proof and individually rational, $\Phi_{i}^{t_{0}}\left(R_{i}^{t_{0}} ; R_{-i}^{t_{0}}\right)=$ $o^{t_{0}}$. Note that since $o^{t_{0}} \in \operatorname{SLC}\left(R_{i}^{\prime t_{0}}, \zeta_{i}^{t_{0}}(R)\right)$, by $(2)$, we have $\zeta_{i}(R) P_{i}^{\prime} \varphi_{i}\left(R_{i}^{\prime} ; R_{-i}\right)$. Thus, by strategy-proofness of $\zeta, \zeta_{i}\left(R_{i}^{\prime} ; R_{-i}\right) P_{i}^{\prime} \varphi_{i}\left(R_{i}^{\prime} ; R_{-i}\right)$. Since $\zeta$ dom $\varphi$, we are done.

Next we present Lemma 3. It tells us how we transit from a profile if the coordinate-wise weak domination does not occur in the profile. Item (lem3-2) is needed to terminate the induction process in Lemma 4 in a finite number of steps, and it is also needed to prove Item (ii) in Lemma 4, which is also a trick to prove that two sequences in the proof of Theorem 1 satisfies (seq-ii). Item (lem3-3) guarantees that the induction argument in Lemma 4 bites. 
To prove Lemma 3, we need a class of preferences. Let $\Sigma\left(T_{i}\right)$ be the set of bijections from $\left\{1, \cdots,\left|T_{i}\right|\right\}$ to $T_{i}$. A preference $R_{i} \in \mathcal{R}\left(X_{i}\right)$ is lexicographic if there are $\sigma \in \Sigma\left(T_{i}\right)$ and list of type preferences $\left(R_{i}^{t}\right)_{t \in T_{i}} \in \prod_{t \in T_{i}} \mathcal{P}\left(X^{t}\right)$ such that for each pair of bundles $\left\{x_{i}, x_{i}^{\prime}\right\} \subseteq X_{i}$ with $x_{i} \neq x_{i}^{\prime}$,

$x_{i} R_{i} x_{i}^{\prime} \Leftrightarrow x_{i}^{\sigma(1)} P_{i}^{\sigma(1)} x_{i}^{\sigma(1)}$ or $\left[\exists t \in\left\{1, \cdots,\left|T_{i}\right|\right\} \backslash\{1\}\right.$ s.t. $\left\{\forall t^{\prime}<t, x_{i}^{\sigma\left(t^{\prime}\right)}=x_{i}^{\prime \sigma\left(t^{\prime}\right)}\right\}$ and $\left.x_{i}^{\sigma(t)} P_{i}^{\sigma(t)} x_{i}^{\sigma(t)}\right]$.

We denote by $\mathcal{P}_{\text {lex }}\left(X_{i}\right)$ the set of all lexicographic preferences on $X_{i}$. It is easy to show that $\mathcal{P}_{\text {lex }}\left(X_{i}\right) \subseteq \mathcal{P}_{\text {add }}\left(X_{i}\right)^{41}$

Lemma 3. Suppose that a rule $\varphi$ is independent and for each $t \in T, \Phi^{t}: \mathcal{P}\left(X^{t}\right)^{N^{t}} \rightarrow \mathcal{A}^{t}$ is strategy-proof. Suppose also that a strategy-proof rule $\zeta$ dominates $\varphi$. Let $R \in \mathcal{D}$. If $R$ does not satisfy the $(\zeta, \varphi)$-reverse property, and not $\zeta(R)$ cw-dom $(R) \varphi(R)$, then there exist $i \in N$ and $R_{i}^{\prime} \in \mathcal{D}_{i}$ such that

(lem3-1) $R_{i} \notin \mathcal{P}\left(X_{i}\right)$,

(lem3-2) $I\left(R_{i}^{\prime} ; R_{-i}\right)<I(R)$ and $B\left(R_{i}^{\prime} ; R_{-i}\right) \leq B(R)$, and

(lem3-3) $\zeta\left(R_{i}^{\prime} ; R_{-i}\right) \operatorname{dom}\left(R_{i}^{\prime} ; R_{-i}\right) \varphi\left(R_{i}^{\prime} ; R_{-i}\right)$

Proof. First, we show that there exists $i \in N$ satisfying (lem3-1). Since not $\zeta(R)$ cw-dom $(R) \varphi(R)$, there exist $i \in N$ and $t_{0} \in T_{i}$ such that $\varphi_{i}^{t_{0}}(R) P_{i}^{t_{0}} \zeta_{i}^{t_{0}}(R)$. Thus, since $R$ does not satisfy the $(\zeta, \varphi)$ reverse property, we have $\varphi_{i}(R) R_{i} \zeta_{i}(R)$. Since $\zeta \operatorname{dom} \varphi, \varphi_{i}(R) I_{i} \zeta_{i}(R)$. Since $\varphi_{i}(R) \neq \zeta_{i}(R)$, we obtain that $R_{i} \notin \mathcal{P}\left(X_{i}\right)$, i.e., (lem3-1).

Next we choose $R_{i}^{\prime} \in \mathcal{D}_{i}$ as follows. By Assumption $2, R_{i} \in R_{a d d}\left(X_{i}\right)$. Let $\left(u_{i}^{t}\right)_{t \in T_{i}} \in \prod_{t \in T_{i}} \mathcal{U}^{t}$ be a list of type-utility functions which represents $R_{i}$. Since $u_{i}^{t_{0}}\left(\varphi_{i}^{t_{0}}(R)\right)>u_{i}^{t_{0}}\left(\zeta_{i}^{t_{0}}(R)\right)$ and $\sum_{t \in T_{i}} u_{i}^{t}\left(\varphi_{i}^{t}(R)\right)=\sum_{t \in T_{i}} u_{i}^{t}\left(\zeta_{i}^{t}(R)\right)$, there exists $t_{1} \in T_{i}$ such that $u_{i}^{t_{1}}\left(\zeta_{i}^{t_{1}}(R)\right)>u_{i}^{t_{1}}\left(\varphi_{i}^{t_{1}}(R)\right)$, i.e.,

Proof of $\mathcal{P}_{\text {lex }}\left(X_{i}\right) \subseteq \mathcal{P}_{\text {add }}\left(X_{i}\right)$. Let $R_{i} \in \mathcal{P}_{\text {lex }}\left(X_{i}\right)$. Suppose that $R_{i}$ is characterized by $\sigma \in \Sigma\left(T_{i}\right)$ and $\left(R_{i}^{t}\right)_{t \in T_{i}} \in$ $\prod_{t \in T_{i}} \mathcal{P}\left(X^{t}\right)$. Assume, without loss of generality, that $T_{i}=T$ and $\sigma$ is the identity mapping, and $\left(R_{i}^{t}\right)_{t \in T}$ is such that

\begin{tabular}{cccc}
$R_{i}^{1}$ & $R_{i}^{2}$ & $\cdots$ & $R_{i}^{|T|}$ \\
\hline$x^{11}$ & $x^{21}$ & & $x^{|T| 1}$ \\
$x^{12}$ & $x^{22}$ & & $x^{|T| 2}$ \\
$\vdots$ & $\vdots$ & $\cdots$ & $\vdots$ \\
$x^{1 K_{1}}$ & $x^{2 K_{2}}$ & & $x^{|T| K_{|T|}}$
\end{tabular}

where for each $t \in T, K_{t}:=\left|X^{t}\right|$. Let $K:=\sum_{t \in T} K_{t}$. Now we define $\left(u_{i}^{t}\right)_{t \in T} \in \Pi_{t \in T} \mathcal{U}^{t}$ as follows:

$$
\begin{array}{cccc}
u_{i}^{1}\left(x^{11}\right)=10^{K-1} & u_{i}^{2}\left(x^{21}\right)=10^{K-K_{1}-1} & & u_{i}^{|T|}\left(x^{|T| 1}\right)=10^{K-\sum_{t=1}^{|T|} K_{t}-1} \\
u_{i}^{1}\left(x^{12}\right)=10^{K-2} & u_{i}^{2}\left(x^{22}\right)=10^{K-K_{1}-2} & & u_{i}^{|T|}\left(x^{|T| 2}\right)=10^{K-\sum_{t=1}^{|T|-1} K_{t}-2} \\
\vdots & \vdots & \ldots & \vdots \\
u_{i}^{1}\left(x^{1 K_{1}}\right)=10^{K-K_{1}} & u_{i}^{2}\left(x^{2 K_{2}}\right)=10^{K-\left(K_{1}+K_{2}\right)} & & u_{i}^{|T|}\left(x^{|T| K_{|T|}}\right)=10^{K-\sum_{t=1}^{|T|} K_{t}} .
\end{array}
$$

Obviously, for each $\left\{y_{i}, z_{i}\right\} \subseteq X_{i}, y_{i} R_{i} z_{i}$ if and only if $\sum_{t \in T} u_{i}^{t}\left(y_{i}^{t}\right) \geq \sum_{t \in T} u_{i}^{t}\left(z_{i}^{t}\right)$. Thus $R_{i} \in \mathcal{P}_{\text {add }}\left(X_{i}\right)$. 
$\zeta_{i}^{t_{1}}(R) P_{i}^{t_{1}} \varphi_{i}^{t_{1}}(R)$. Now we change the preference of agent $i$. Let $\left(R_{i}^{\prime t}\right)_{t \in T_{i}}:=\left(R_{i}^{t}\right)_{t \in T_{i}}$ and $\sigma \in \Sigma\left(T_{i}\right)$ be such that $\sigma(1)=t_{1}$. Let $R_{i}^{\prime} \in \mathcal{P}_{\text {lex }}\left(X_{i}\right)$ be the lexicographic preference defined by $\sigma$ and $\left(R_{i}^{\prime t}\right)_{t \in T_{i}}$ (See Remark 1). Obviously, $I\left(R_{i}^{\prime} ; R_{-i}\right)<I(R)$ and $B\left(R_{i}^{\prime} ; R_{-i}\right)=B(R)$. Thus, Item (3-2) is satisfied.

Finally we show (lem3-3). First, since $\varphi$ is independent and $R_{i}^{t_{1}}=R_{i}^{t_{1}}, \varphi_{i}^{t_{1}}\left(R_{i}^{\prime} ; R_{-i}\right)=\varphi_{i}^{t_{1}}(R)$. Second, since $\zeta$ is strategy-proof, $\zeta_{i}\left(R_{i}^{\prime} ; R_{-i}\right) R_{i}^{\prime} \zeta_{i}(R)$. Third, since $R_{i}^{\prime}$ is a lexicographic preference whose first priority is assigned to $t_{1}$, we have $\zeta_{i}^{t_{1}}\left(R_{i}^{\prime} ; R_{-i}\right) R_{i}^{\prime t_{1}} \zeta_{i}^{t_{1}}(R)$. Therefore, since $\zeta_{i}^{t_{1}}(R) P_{i}^{t_{1}}$ $\varphi_{i}^{t_{1}}(R)$ and $R_{i}^{\prime t_{1}}=R_{i}^{t_{1}}$, we have $\zeta_{i}^{t_{1}}\left(R_{i}^{\prime} ; R_{-i}\right) R_{i}^{\prime t_{1}} \zeta_{i}^{t_{1}}(R) P_{i}^{\prime t_{1}} \varphi_{i}^{t_{1}}(R)=\varphi_{i}^{t_{1}}\left(R_{i}^{\prime} ; R_{-i}\right)$. Since $R_{i}^{\prime}$ is a lexicographic preference whose first priority is on $t_{1}, \zeta_{i}\left(R_{i}^{\prime} ; R_{-i}\right) P_{i}^{\prime} \varphi_{i}\left(R_{i}^{\prime} ; R_{-i}\right)$. Since $\zeta$ dom $\varphi$, we are done.

The following lemma is the realization of the transition process we employ. Item (i) is the trick we need when we prove the two resulting sequences of non-negative integers in the proof of Theorem 1 meet the condition (seq-ii). Item (ii) is also a trick used to prove the condition (seq-ii). Item (iii) asserts that the transition process finally reaches a profile which satisfies the $(\zeta, \varphi)$-reverse property.

Lemma 4. Suppose that a rule $\varphi$ is independent and for each $t \in T, \Phi^{t}: \mathcal{P}\left(X^{t}\right)^{N^{t}} \rightarrow \mathcal{A}^{t}$ is strategyproof and non-wasteful. Suppose also that a strategy-proof rule $\zeta$ dominates $\varphi$. Let $R^{(0)} \in \mathcal{D}$ be such that $\zeta\left(R^{(0)}\right) \operatorname{dom}\left(R^{0}\right) \varphi\left(R^{(0)}\right)$. If $R^{(0)}$ does not satisfy the $(\zeta, \varphi)$-reverse property, then there exists a finite sequence of agent-preference pairs $\left\{\left(j^{(\ell)}, R_{j^{(\ell)}}^{(\ell)}\right\}_{\ell=1}^{L}\right.$ satisfying the following conditions (i), (ii) and (iii). For each $\ell=1, \cdots, L$, let $R^{(\ell)}:=\left(R_{j^{(\ell)}}^{(\ell)} ; R_{-j^{(\ell)}}^{(\ell-1)}\right)$.

(i) $\forall \ell=1, \cdots, L,\left[\left\{\exists t \in T_{j^{(\ell)}}\right.\right.$ s.t. $\left.\left|S U C\left(R_{j^{(\ell)}}^{(\ell-1) t}, o^{t}\right)\right| \geq 2\right\}$ or $\left.R_{j^{(\ell)}}^{(\ell-1)} \notin \mathcal{P}\left(X_{j^{(\ell)}}\right)\right]$

(ii) $B\left(R^{(0)}\right) \geq B\left(R^{(1)}\right) \geq \cdots \geq B\left(R^{(L)}\right)$, and

(iii) $R^{(L)}$ satisfies the $(\zeta, \varphi)$-reverse property.

Proof. We inductively construct a sequence.

Step 1: If $\zeta\left(R^{(0)}\right) \mathrm{cw}-\operatorname{dom}\left(R^{(0)}\right) \varphi\left(R^{(0)}\right)$, then by Lemma 2, there exist $j^{(1)} \in N$ and $R_{j^{(1)}}^{(1)} \in \mathcal{D}_{j^{(1)}}$ satisfying (lem2-1), (lem2-2) and (lem2-3). If not, then by Lemma 3, there exist $j^{(1)} \in N$ and $R_{j^{(1)}}^{(1)} \in \mathcal{D}_{j^{(1)}}$ satisfying (lem3-1), (lem3-2) and (lem3-3). Then, by (lem2-1) or (lem3-1), \{there is $t \in T_{j^{(1)}}$ s.t. $\left.\left|\operatorname{SUC}\left(R_{j^{(1)}}^{(0) t}, o^{t}\right)\right| \geq 2\right\}$ or $R_{j^{(1)}}^{(0)} \notin \mathcal{P}\left(X_{j^{(1)}}\right)$. Moreover, by (lem2-2) or (lem3-2), $B\left(R^{(0)}\right) \geq B\left(R^{(1)}\right)$. Therefore, if $R^{(1)}$ satisfies the $(\zeta, \varphi)$-reverse property, then $\left(j^{(1)}, R_{j^{(1)}}^{(1)}\right)$ is the desired sequence with its length 1 . If $R^{(1)}$ does not satisfy the $(\zeta, \varphi)$-reverse property, then go to the next step. Note that by (lem2-3) or (lem3-3), $\zeta\left(R^{(1)}\right) \operatorname{dom}\left(R^{(1)}\right) \varphi\left(R^{(1)}\right) .{ }^{42}$

Step $\ell(\geq 2)$ : If $\zeta\left(R^{(\ell-1)}\right) \operatorname{cw}-\operatorname{dom}\left(R^{(\ell-1)}\right) \varphi\left(R^{(\ell-1)}\right)$, then by Lemma 2 , there exist $j^{(\ell)} \in N$ and $R_{j^{(\ell)}}^{(\ell)} \in \mathcal{D}_{j^{(\ell)}}$ satisfying (lem2-1), (lem2-2) and (lem2-3). If not, then by Lemma 3 , there exist $j^{(\ell)} \in N$ and $R_{j^{(\ell)}}^{(\ell)} \in \mathcal{D}_{j^{(\ell)}}$ satisfying (lem3-1), (lem3-2) and (lem3-3). Then, by (lem2-1) or (lem3-1), $\left\{\right.$ there is $t \in T_{j^{(\ell)}}$ s.t. $\left.\left|\mathrm{SUC}\left(R_{j^{(\ell)}}^{(\ell-1) t}, o^{t}\right)\right| \geq 2\right\}$ or $R_{j^{(\ell)}}^{(\ell-1)} \notin \mathcal{P}\left(X_{j^{(\ell)}}\right)$. Moreover, by (lem2-2) or (lem3-2), $B\left(R^{(\ell-1)}\right) \geq B\left(R^{(\ell)}\right)$. Therefore, if $R^{(\ell)}$ satisfies the $(\zeta, \varphi)$-reverse property,

\footnotetext{
${ }^{42}$ Therefore, the induction argument bites.
} 
then $\left(j^{(1)}, R_{j^{(1)}}^{(1)}\right), \cdots,\left(j^{(\ell)}, R_{j^{(\ell)}}^{(\ell)}\right)$ is the desired sequence with its length $\ell$. If $R^{(\ell)}$ does not satisfy the $(\zeta, \varphi)$-reverse property, then go to the next step. Note that by (lem2-3) or (lem3-3), $\zeta\left(R^{(\ell)}\right) \operatorname{dom}\left(R^{(\ell)}\right) \varphi\left(R^{(\ell)}\right)$.

We claim that the above procedure stops in a finite number of steps, i.e., there exists $L \geq 1$ such that $R^{(L)}$ satisfies the $(\zeta, \varphi)$-reverse property. Since in each step $\ell$, by (lem2-2) or (lem3-2),

$$
\left[B\left(R^{(\ell-1)}\right) \geq B\left(R^{(\ell)}\right) \text { and } I\left(R^{(\ell-1)}\right) \geq I\left(R^{(\ell)}\right)\right] \text { and }\left[B\left(R^{(\ell-1)}\right)>B\left(R^{(\ell)}\right) \text { or } I\left(R^{(\ell-1)}\right)>I\left(R^{(\ell)}\right)\right] \text {. }
$$

Since $B\left(R^{(0)}\right)$ and $I\left(R^{(0)}\right)$ are non-negative integers, the procedure cannot have infinite steps.

\section{Proof of Theorem 1}

Suppose to the contrary that a strategy-proof rule $\zeta$ dominates $\varphi$. Let $R^{(1,0)} \in \mathcal{D}$ be such that $\zeta\left(R^{(1,0)}\right) \operatorname{dom}\left(R^{(1,0)}\right) \varphi\left(R^{(1,0)}\right)$. Let $b^{(1,0)}:=\zeta\left(R^{(1,0)}\right)$ and $a^{(1,0)}:=\varphi\left(R^{(1,0)}\right)$. Let $N^{(0)}:=|N|$ and $B^{(1)}:=B\left(R^{(1,0)}\right)$. We will inductively construct two infinite sequences of non-negative integers $\left\{N^{(k)}\right\}_{k=0}^{\infty}$ and $\left\{B^{(k)}\right\}_{k=1}^{\infty}$ that cause a contradiction.

Induction step 1. If $R^{(1,0)}$ satisfies the $(\zeta, \varphi)$-reverse property, then let $L_{1}:=0$. If $R^{(1,0)}$ does not, then by Lemma 4 , there exists a finite sequence of agent-preference pairs $\left\{\left(j^{(1, \ell)}, R_{j^{(1, \ell)}}^{(1, \ell)}\right)\right\}_{\ell=1}^{L_{1}}$ satisfying the following (1-i), (1-ii) and (1-iii), where for each $\ell \in\left\{1, \cdots, L_{1}\right\}, R^{(1, \ell)}:=\left(R_{j^{(1, \ell)}}^{(1, \ell)} ; R_{-j^{(1, \ell)}}^{(1, \ell-1)}\right)$.

(1-i) $\forall \ell=1, \cdots, L_{1},\left[\left\{\exists t \in T_{j^{(1, \ell)}}\right.\right.$ s.t. $\left.\left|\operatorname{SUC}\left(R_{j^{(1, \ell)}}^{(1, \ell-1) t}, o^{t}\right)\right| \geq 2\right\}$ or $\left.R_{j^{(1, \ell)}}^{(1, \ell-1)} \notin \mathcal{P}\left(X_{j^{(1, \ell)}}\right)\right]$,

(1-ii) $B\left(R^{(1,0)}\right) \geq B\left(R^{(1,1)}\right) \geq \cdots \geq B\left(R^{\left(1, L_{1}\right)}\right)$, and

(1-iii) $R^{\left(1, L_{1}\right)}$ satisfies the $(\zeta, \varphi)$-reverse property.

For each $\ell \in\left\{1, \cdots, L_{1}\right\}$, let $b^{(1, \ell)}:=\zeta\left(R^{(1, \ell)}\right)$ and $a^{(1, \ell)}:=\varphi\left(R^{(1, \ell)}\right)$.

Since $R^{\left(1, L_{1}\right)}$ satisfies the $(\zeta, \varphi)$-reverse property,

$$
\exists i^{(1)} \in N \text { s.t. }\left[b_{i^{(1)}}^{\left(1, L_{1}\right)} P_{i^{(1)}}^{\left(1, L_{1}\right)} a_{i^{(1)}}^{\left(1, L_{1}\right)} \text { and }\left\{\exists t \in T_{i^{(1)}} \text { s.t. } a_{i^{(1)}}^{\left(1, L_{1}\right) t} P_{i^{(1)}}^{\left(1, L_{1}\right) t} b_{i^{(1)}}^{\left(1, L_{1}\right) t}\right\}\right] .
$$

Let $N^{(1)}:=\left|N \backslash\left\{i^{(1)}\right\}\right|$. Now, we change the agent $i^{(1)}$ 's preference. Let $\left(\tilde{R}_{i^{(1)}}^{(2,0) t}\right)_{t \in T_{i(1)}}$ be a list of type preferences satisfying the $\left(1^{*}-i\right)$ below. By applying Lemma 1 for $\left(\tilde{R}_{i^{(1)}}^{(2,0) t}\right)_{t \in T_{i(1)}}$ and $\left(o^{t}\right)_{t \in T_{i(1)}}$, we obtain a preference $R_{i^{(1)}}^{(2,0)} \in \mathcal{P}_{a d d}\left(X_{i^{(1)}}\right)$ with $\left(R_{i^{(1)}}^{(2,0) t}\right)_{t \in T_{i(1)}}=\left(\tilde{R}_{i^{(1)}}^{(2,0) t}\right)_{t \in T_{i(1)}}$ satisfying $\left(1^{*}-i i\right)$.

$\left(\mathbf{1}^{*} \mathbf{- i}\right) \forall t \in T_{i^{(1)}}, \forall x^{t} \in X^{t} \backslash\left\{b_{i^{(1)}}^{\left(1, L_{1}\right) t}, o^{t}\right\}, b_{i^{(1)}}^{\left(1, L_{1}\right) t} \tilde{R}_{i^{(1)}}^{(2,0) t} o^{t} \tilde{P}_{i^{(1)}}^{(2,0) t} x^{t}$ and

(1*-ii) $\forall x_{i^{(1)}} \in X_{i^{(1)}},\left[\left\{\exists t \in T_{i^{(1)}}\right.\right.$ s.t. $\left.\left.x_{i^{(1)}}^{t} \in \operatorname{SLC}\left(R_{i^{(1)}}^{(2,0) t}, o^{t}\right)\right\} \Rightarrow\left(o^{t}\right)_{t \in T_{i}(1)} P_{i^{(1)}}^{(2,0)} x_{i^{(1)}}\right]$.

Let $R^{(2,0)}:=\left(R_{i^{(1)}}^{(2,0)} ; R_{-i^{(1)}}^{\left(1, L_{1}\right)}\right), b^{(2,0)}:=\zeta\left(R^{(2,0)}\right), a^{(2,0)}:=\varphi\left(R^{(2,0)}\right)$ and $B^{(2)}:=B\left(R^{(2,0)}\right)$.

Claim 1.1. $b^{(2,0)} \operatorname{dom}\left(R^{(2,0)}\right) a^{(2,0)}$.

We show $b_{i^{(1)}}^{(2,0)} P_{i^{(1)}}^{(2,0)} a_{i^{(1)}}^{(2,0)}$. Suppose to the contrary that $b_{i^{(1)}}^{(2,0)}=a_{i^{(1)}}^{(2,0)}$. 43 Since $\zeta$ is strategyproof, $b_{i^{(1)}}^{(2,0)}=\zeta_{i^{(1)}}\left(R^{(2,0)}\right) R_{i^{(1)}}^{(2,0)} \zeta_{i^{(1)}}\left(R^{\left(1, L_{1}\right)}\right)=b_{i^{(1)}}^{\left(1, L_{1}\right)}$. Note that by $\left(1^{*}-\mathrm{i}\right)$ and the separability

${ }^{43}$ Note that $R_{i^{(1)}}^{(2,0)}$ is strict. 
of $R_{i^{(1)}}^{(2,0)}, b_{i^{(1)}}^{\left(1, L_{1}\right)}$ is a best bundle at $R_{i^{(1)}}^{(2,0)}$. Thus, since $R_{i^{(1)}}^{(2,0)}$ is strict, $b_{i^{(1)}}^{(2,0)}=b_{i^{(1)}}^{\left(1, L_{1}\right)}$. Thus, $a_{i^{(1)}}^{(2,0)}=b_{i^{(1)}}^{\left(1, L_{1}\right)}$. Hence, by $\mathrm{A}(1), \varphi_{i^{(1)}}\left(R^{(2,0)}\right)=a_{i^{(1)}}^{(2,0)}=b_{i^{(1)}}^{\left(1, L_{1}\right)} P_{i^{(1)}}^{\left(1, L_{1}\right)} a_{i^{(1)}}^{\left(1, L_{1}\right)}=\varphi_{i^{(1)}}\left(R^{\left(1, L_{1}\right)}\right)$, a violation of strategy-proofness of $\varphi$. Therefore, $b_{i^{(1)}}^{(2,0)} P_{i^{(1)}}^{(2,0)} a_{i^{(1)}}^{(2,0)}$. Since $\zeta \operatorname{dom} \varphi$, this completes the proof of Claim 1.1.

The following claim trivially holds since $N^{(0)}>N^{(1)}$.

Claim 1.2. $N^{(0)}=N^{(1)} \Rightarrow B^{(1)}>B^{(2)}$.

Now, let $k \geq 2$.

Induction hypothesis. Suppose that the series of statements below are true for each $k^{\prime}<k$. If $\left\{\left(j^{\left(k^{\prime}, \ell\right)}, R_{j^{\left(k^{\prime}, \ell\right)}}^{\left(k^{\prime}, \ell\right)}\right)\right\}_{\ell=1}^{L_{k^{\prime}}}$ is not defined, $L_{k^{\prime}}=0$. If $\left\{\left(j^{\left(k^{\prime}, \ell\right)}, R_{j^{\left(k^{\prime}, \ell\right)}}^{\left(k^{\prime}, \ell\right)}\right\}_{\ell=1}^{L_{k^{\prime}}}\right.$ is defined, then it satisfies the following (k'-i), (k'-ii) and (k'-iii), where for each $\ell \in\left\{1, \cdots, L_{k^{\prime}}\right\}, R^{\left(k^{\prime}, \ell\right)}:=\left(R_{j\left(k^{\prime}, \ell\right)}^{\left(k^{\prime}, \ell\right)} ; R_{-j\left(k^{\prime}, \ell\right)}^{\left(k^{\prime}, \ell-1\right)}\right)$.

(k'-i) $\forall \ell=1, \cdots, L_{k^{\prime}},\left[\left\{\exists t \in T_{j^{\left(k^{\prime}, \ell\right)}}\right.\right.$ s.t. $\left.\left|\operatorname{SUC}\left(R_{j^{\left(k^{\prime}, \ell\right)}}^{\left(k^{\prime}, \ell-1\right) t}, o^{t}\right)\right| \geq 2\right\}$ or $\left.R_{j^{\left(k^{\prime}, \ell\right)}}^{\left(k^{\prime}, \ell-1\right)} \notin \mathcal{P}\left(X_{j^{\left(k^{\prime}, \ell\right)}}\right)\right]$,

(k'-ii) $B\left(R^{\left(k^{\prime}, 0\right)}\right) \geq B\left(R^{\left(k^{\prime}, 1\right)}\right) \geq \cdots \geq B\left(R^{\left(k^{\prime}, L_{k^{\prime}}\right)}\right)$, and

(k'-iii) $R^{\left(k^{\prime}, L_{k^{\prime}}\right)}$ satisfies the $(\zeta, \varphi)$-reverse property.

For each $\ell=1, \cdots, L_{k^{\prime}}$, let $b^{\left(k^{\prime}, \ell\right)}:=\zeta\left(R^{\left(k^{\prime}, \ell\right)}\right)$ and $a^{\left(k^{\prime}, \ell\right)}:=\varphi\left(R^{\left(k^{\prime}, \ell\right)}\right)$. For agent $i^{\left(k^{\prime}\right)} \in N$,

$$
\left[b_{i^{\left(k^{\prime}\right)}}^{\left(k^{\prime}, L_{k^{\prime}}\right)} P_{i^{\left(k^{\prime}\right)}}^{\left(k^{\prime}, L_{k^{\prime}}\right)} a_{i^{\left(k^{\prime}\right)}}^{\left(k^{\prime}, L_{k^{\prime}}\right)} \text { and }\left\{\exists t \in T_{i\left(k^{\prime}\right)} \text { s.t. } a_{i^{\left(k^{\prime}\right)}}^{\left(k^{\prime}, L_{k^{\prime}}\right) t} P_{i^{\left(k^{\prime}\right)}}^{\left(k^{\prime}, L_{k^{\prime}}\right) t} b_{i^{\left(k^{\prime}\right)}}^{\left(k^{\prime}, L_{k^{\prime}}\right) t}\right\}\right]
$$

Let $N^{\left(k^{\prime}\right)}:=\left|N \backslash\left\{i^{(1)}, \cdots, i^{\left(k^{\prime}\right)}\right\}\right|$. For agent $i^{\left(k^{\prime}\right)}$ 's new preference $R_{i^{\left(k^{\prime}\right)}}^{\left(k^{\prime}+1,0\right)} \in \mathcal{P}_{a d d}\left(X_{i\left(k^{\prime}\right)}\right)$,

$\left(\mathbf{k}^{9^{*}}\right.$-i) $\forall t \in T_{i^{\left(k^{\prime}\right)}}, \forall x^{t} \in X^{t} \backslash\left\{b_{i^{\left(k^{\prime}\right)}}^{\left(k^{\prime}, L_{k^{\prime}}\right) t}, o^{t}\right\}, b_{i^{\left(k^{\prime}\right)}}^{\left(k^{\prime}, L_{k^{\prime}}\right) t} R_{i^{\left(k^{\prime}\right)}}^{\left(k^{\prime}+1,0\right) t} o^{t} P_{i^{\left(k^{\prime}\right)}}^{\left(k^{\prime}+1,0\right) t} x^{t}$, and

$\left(\mathbf{k}^{*}\right.$-ii) $\forall x_{i^{\left(k^{\prime}\right)}} \in X_{i\left(k^{\prime}\right)},\left[\left\{\exists t \in T_{i^{\left(k^{\prime}\right)}}\right.\right.$ s.t. $\left.\left.x_{i\left(k^{\prime}\right)}^{t} \in \operatorname{SLC}\left(R_{i\left(k^{\prime}\right)}^{\left(k^{\prime}+1,0\right) t}, o^{t}\right)\right\} \Rightarrow\left(o^{t}\right)_{t \in T_{i\left(k^{\prime}\right)}} P_{i\left(k^{\prime}\right)}^{\left(k^{\prime}+1,0\right)} x_{i\left(k^{\prime}\right)}\right]$.

Letting $R^{\left(k^{\prime}+1,0\right)}:=\left(R_{i^{\left(k^{\prime}\right)}}^{\left(k^{\prime}+1,0\right)} ; R_{-i^{\left(k^{\prime}\right)}}^{\left(k^{\prime}, L_{k^{\prime}}\right)}\right), b^{\left(k^{\prime}+1,0\right)}:=\zeta\left(R^{\left(k^{\prime}+1,0\right)}\right), a^{\left(k^{\prime}+1,0\right)}:=\varphi\left(R^{\left(k^{\prime}+1,0\right)}\right)$ and $B^{\left(k^{\prime}+1\right)}:=$ $B\left(R^{\left(k^{\prime}+1,0\right)}\right)$.

Claim k'.1. $b^{\left(k^{\prime}+1,0\right)} \operatorname{dom}\left(R^{\left(k^{\prime}+1,0\right)}\right) a^{\left(k^{\prime}+1,0\right)}$, and

Claim k'.2. $N^{\left(k^{\prime}-1\right)}=N^{\left(k^{\prime}\right)} \Rightarrow B^{\left(k^{\prime}\right)}>B^{\left(k^{\prime}+1\right)}$.

Induction step $\boldsymbol{k}$. If $R^{(k, 0)}$ satisfies the $(\zeta, \varphi)$-reverse property, then let $L_{k}:=0$. If $R^{(k, 0)}$ does not, then by Lemma 4 , there exists a finite sequence of agent-preference pairs $\left\{\left(j^{(k, \ell)}, R_{j^{(k, \ell)}}^{(k, \ell)}\right)\right\}_{\ell=1}^{L_{k}}$ satisfying the following $(\mathrm{k}-\mathrm{i})$, (k-ii) and (k-iii), where for each $\ell=1, \cdots, L_{k}, R^{(k, \ell)}:=\left(R_{j^{(k, \ell)}}^{(k, \ell)} ; R_{-j(k, \ell)}^{(k, \ell-1)}\right)$.

(k-i) $\forall \ell=1, \cdots, L_{k},\left[\left\{\exists t \in T_{j^{(k, \ell)}}\right.\right.$ s.t. $\left.\left|\operatorname{SUC}\left(R_{j^{(k, \ell)}}^{(k, \ell-1) t}, o^{t}\right)\right| \geq 2\right\}$ or $\left.R_{j^{(k, \ell)}}^{(k, \ell-1)} \notin \mathcal{P}\left(X_{j^{(k, \ell)}}\right)\right]$,

(k-ii) $B\left(R^{(k, 0)}\right) \geq B\left(R^{(k, 1)}\right) \geq \cdots \geq B\left(R^{\left(k, L_{k}\right)}\right)$, and

(k-iii) $R^{\left(k, L_{k}\right)}$ satisfies the $(\zeta, \varphi)$-reverse property.

For each $\ell=1, \cdots, L_{k}$, let $b^{(k, \ell)}:=\zeta\left(R^{(k, \ell)}\right)$ and $a^{(k, \ell)}:=\varphi\left(R^{(k, \ell)}\right)$. Since $R^{\left(k, L_{k}\right)}$ satisfies the $(\zeta, \varphi)$-reverse property,

$$
\exists i^{(k)} \in N \text { s.t. }\left[b_{i^{(k)}}^{\left(k, L_{k}\right)} P_{i^{(k)}}^{\left(k, L_{k}\right)} a_{i^{(k)}}^{\left(k, L_{k}\right)} \text { and }\left\{\exists t \in T_{i^{(k)}} \text { s.t. } a_{i^{(k)}}^{\left(k, L_{k}\right) t} P_{i^{(k)}}^{\left(k, L_{k}\right) t} b_{i^{(k)}}^{\left(k, L_{k}\right) t}\right\}\right] .
$$


Let $N^{(k)}:=\left|N \backslash\left\{i^{(1)}, \cdots, i^{(k)}\right\}\right|$. Now, we change the agent $i^{(k)}$ 's preference. Let $\left(\tilde{R}_{i^{(k)}}^{(k+1,0) t}\right)_{t \in T_{i(k)}}$ be a list of type preferences satisfying the $\left(k^{*}-i\right)$ below. By applying Lemma 1 for $\left(\tilde{R}_{i^{(k)}}^{(k+1,0) t}\right)_{t \in T_{i(k)}}$ and $\left(o^{t}\right)_{t \in T_{i(k)}}$, we obtain a preference $R_{i^{(k)}}^{(k+1,0)} \in \mathcal{P}_{\text {add }}\left(X_{i(k)}\right)$ with $\left(R_{i^{(k)}}^{(k+1,0) t}\right)_{t \in T_{i}(k)}=\left(\tilde{R}_{i^{(k)}}^{(k+1,0) t}\right)_{t \in T_{i^{(k)}}}$ satisfying $\left(k^{*}-i i\right)$.

$\left(\mathbf{k}^{*}-\mathbf{i}\right) \forall t \in T_{i^{(k)}}, \forall x^{t} \in X^{t} \backslash\left\{b_{i^{(k)}}^{\left(k, L_{k}\right) t}, o^{t}\right\}, b_{i^{(k)}}^{\left(k, L_{k}\right) t} \tilde{R}_{i^{(k)}}^{(k+1,0) t} o^{t} \tilde{P}_{i^{(k)}}^{(k+1,0) t} x^{t}$ and

(k $\mathbf{k}^{*}$-ii) $\forall x_{i^{(k)}} \in X_{i^{(k)}},\left[\left\{\exists t \in T_{i^{(k)}}\right.\right.$ s.t. $\left.\left.x_{i^{(k)}}^{t} \in \operatorname{SLC}\left(R_{i^{(k)}}^{(k+1,0) t}, o^{t}\right)\right\} \Rightarrow\left(o^{t}\right)_{t \in T_{i(k)}} P_{i^{(k)}}^{(k+1,0)} x_{i^{(k)}}\right]$.

Let $R^{(k+1,0)}:=\left(R_{i^{(k)}}^{(k+1,0)} ; R_{-i^{(k)}}^{\left(k, L_{k}\right)}\right), b^{(k+1,0)}:=\zeta\left(R^{(k+1,0)}\right), a^{(k+1,0)}:=\varphi\left(R^{(k+1,0)}\right)$ and $B^{(k+1)}:=$ $B\left(R^{(k+1,0)}\right)$.

Claim k.1. $b^{(k+1,0)} \operatorname{dom}\left(R^{(k+1,0)}\right) a^{(k+1,0)}$.

We show that $b_{i^{(k)}}^{(k+1,0)} P_{i^{(k)}}^{(k+1,0)} a_{i^{(k)}}^{(k+1,0)}$. Suppose to the contrary that $b_{i^{(k)}}^{(k+1,0)}=a_{i^{(k)}}^{(k+1,0)}$. ${ }^{44}$ Since $\zeta$ is strategy-proof, $b_{i^{(k)}}^{(k+1,0)}=\zeta_{i^{(k)}}\left(R^{(k+1,0)}\right) R_{i^{(k)}}^{(k+1,0)} \zeta_{i^{(k)}}\left(R^{\left(k, L_{k}\right)}\right)=b_{i^{(k)}}^{\left(k, L_{k}\right)}$. Note that by $\left(\mathrm{k}^{*}-\mathrm{i}\right)$ and the separability of $R_{i^{(k)}}^{(k+1,0)}, b_{i(k)}^{\left(k, L_{k}\right)}$ is a best bundle at $R_{i^{(k)}}^{(k+1,0)}$. Since $R_{i^{(k)}}^{(k+1,0)}$ is strict, $b_{i^{(k)}}^{(k+1,0)}=b_{i^{(k)}}^{\left(k, L_{k}\right)}$. Thus, $a_{i^{(k)}}^{(k+1,0)}=b_{i^{(k)}}^{\left(k, L_{k}\right)}$. Hence, by A $(\mathrm{k}), \varphi_{i^{(k)}}\left(R^{(k+1,0)}\right)=a_{i^{(k)}}^{(k+1,0)}=b_{i^{(k)}}^{\left(k, L_{k}\right)} P_{i^{(k)}}^{\left(k, L_{k}\right)}$ $a_{i^{(k)}}^{\left(k, L_{k}\right)}=\varphi_{i^{(k)}}\left(R^{\left(k, L_{k}\right)}\right)$, a violation of strategy-proofness of $\varphi$. Therefore, $b_{i^{(k)}}^{(k+1,0)} P_{i^{(k)}}^{(k+1,0)} a_{i^{(k)}}^{(k+1,0)}$. Since $\zeta \operatorname{dom} \varphi$, this completes the proof of Claim k.1.

Claim k.2. $N^{(k-1)}=N^{(k)} \Rightarrow B^{(k)}>B^{(k+1)}$.

Suppose that $N^{(k-1)}=N^{(k)}$, i.e., $i^{(k)} \in\left\{i^{(1)}, \cdots, i^{(k-1)}\right\}$. Let $k^{\prime}$ be the largest integer with $k^{\prime}<k$ such that $i^{\left(k^{\prime}\right)}=i^{(k)}$. First, we claim that the preference replacement from $R_{i(k)}^{\left(k, L_{k}\right)}$ to $R_{i(k)}^{(k+1,0)}$ is the first opportunity to change $i^{(k)}\left(=i^{\left(k^{\prime}\right)}\right)$ 's preference after we have chosen $R_{i^{\left(k^{\prime}\right)}}^{\left(k^{\prime}+1,0\right)}$. Formally,

Claim k.2.1. $R_{i^{(k)}}^{\left(k, L_{k}\right)}=R_{i^{\left(k^{\prime}\right)}}^{\left(k^{\prime}+1,0\right)}$.

Suppose $R_{i(k)}^{\left(k, L_{k}\right)} \neq R_{i^{\left(k^{\prime}\right)}}^{\left(k^{\prime}+1,0\right)}$. By definition of $k^{\prime}$,

$$
\exists k^{\prime \prime} \in\left\{k^{\prime}+1, \cdots, k\right\}, \exists \ell \in\left\{1, \cdots, L_{k^{\prime \prime}}\right\} \text { s.t. } i^{\left(k^{\prime}\right)}=j^{\left(k^{\prime \prime}, \ell\right)} .{ }^{45}
$$

Let $\left(k^{\prime \prime}, \ell\right)$ be the first index in which $i^{\left(k^{\prime}\right)}=j^{\left(k^{\prime \prime}, \ell\right)} .{ }^{46}$ However, by the condition $(\mathrm{k} "-\mathrm{i})$,

$$
\left\{\exists t \in T_{j^{\left(k^{\prime \prime}, \ell\right)}} \text { s.t. }\left|\mathrm{SUC}\left(R_{j^{\left(k^{\prime \prime}, \ell\right)}}^{\left(k^{\prime \prime}, \ell-1\right) t}, o^{t}\right)\right| \geq 2\right\} \text { or } R_{j^{\left(k^{\prime \prime}, \ell\right)}}^{\left(k^{\prime \prime}, \ell-1\right)} \notin \mathcal{P}\left(X_{j^{\left(k^{\prime \prime}, \ell\right)}}\right)
$$

while $R_{i\left(k^{\prime}\right)}^{\left(k^{\prime}+1,0\right)}\left(=R_{j^{\left(k^{\prime \prime}, \ell\right)}}^{\left(k^{\prime \prime}, \ell-1\right)}\right)$ belongs to $\mathcal{P}\left(X_{i\left(k^{\prime}\right)}\right)$, and satisfies the condition $\left(\mathrm{k}^{\prime *}-\mathrm{i}\right)$ which asserts that for each $t \in T_{i\left(k^{\prime}\right)},\left|\operatorname{SUC}\left(R_{i^{\left(k^{\prime}\right)}}^{\left(k^{\prime}+1,0\right) t}, o^{t}\right)\right| \leq 1$, a contradiction. This completes the proof of Claim k.2.1.

The next claim asserts that for each $t \in T_{i(k)}$, the type- $t$ obeject at $b_{i(k)}^{\left(k, L_{k}\right)}$ is at least as good as the type- $t$ outside option for agent $i^{(k)}$ according to $R_{i^{(k)}}^{\left(k, L_{k}\right)}$.

\footnotetext{
${ }^{44}$ Note that $R_{i^{(k)}}^{(k+1,0)}$ is strict.

${ }^{45}$ In words, the preference replacement is accompanied by the application of Lemma 4 in step $k^{\prime \prime}$ after $k^{\prime}$.

${ }^{46}$ Namely, $k^{\prime \prime}$ is the smallest first coordinate among the pairs satisfying the condition, and $\ell$ is the smallest second coordinate among the pairs in which the first coordinate is $k^{\prime \prime}$ satisfying the condition.
} 


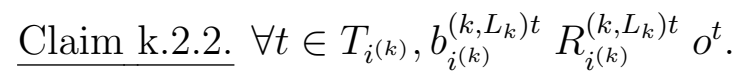

Suppose to the contrary that there exists $t \in T_{i^{(k)}}$ such that $o^{t} P_{i^{(k)}}^{\left(k, L_{k}\right) t} b_{i^{(k)}}^{\left(k, L_{k}\right) t}$. By Claim k.2.1., $R_{i(k)}^{\left(k, L_{k}\right)}=R_{\left.i^{\prime}\right)}^{\left(k^{\prime}+1,0\right)}$. Thus, by the condition $\left(\mathrm{k}^{\prime *}-\mathrm{ii}\right),\left(o^{t}\right)_{t \in T_{i(k)}} P_{i^{(k)}}^{\left(k, L_{k}\right)}$ $b_{i(k)}^{\left(k, L_{k}\right)}=\zeta_{i^{(k)}}\left(R^{\left(k, L_{k}\right)}\right)$. Thus, $\zeta$ is not individually rational. However, since $\zeta \operatorname{dom} \varphi$, $\zeta$ is individually rational, which is a contradiction. This completes the proof of Claim k.2.2.

Now, we turn back to the proof of Claim k.2. By the condition $\mathrm{A}(\mathrm{k})$ and Claim k.2.2., there is $t \in T_{i^{(k)}}$ such that $a_{i^{(k)}}^{\left(k, L_{k}\right) t} P_{i^{(k)}}^{\left(k, L_{k}\right) t} b_{i^{(k)}}^{\left(k, L_{k}\right) t} R_{i^{(k)}}^{\left(k, L_{k}\right) t} o^{t}$. Thus, $B\left(R_{i^{(k)}}^{\left(k, L_{k}\right) t}\right)>B\left(R_{i^{(k)}}^{(k+1,0) t}\right)$. Note that by $\left(\mathrm{k}^{*}-1\right)$ and Claim k.2.2., for each $t^{\prime} \in T_{i(k)} \backslash\{t\}, B\left(R_{i^{(k)}}^{\left(k, L_{k}\right) t^{\prime}}\right) \geq B\left(R_{i^{(k)}}^{(k+1,0) t^{\prime}}\right)$. Summing up, we have $B\left(R_{i^{(k)}}^{\left(k, L_{k}\right)}\right)>B\left(R_{i^{(k)}}^{(k+1,0)}\right)$. Thus, $B\left(R^{\left(k, L_{k}\right)}\right)>B\left(R^{(k+1,0)}\right)$ since $i^{(k)}$ 's preference is the only difference between $R^{\left(k, L_{k}\right)}$ and $R^{(k+1,0)}$. By the condition $(\mathrm{k}-\mathrm{ii}), B\left(R^{(k, 0)}\right) \geq \cdots \geq B\left(R^{\left(k, L_{k}\right)}\right)$. Therefore, $B^{(k)}=B\left(R^{(k, 0)}\right)>B\left(R^{(k+1,0)}\right)=B^{(k+1)}$. This completes the proof of Claim k.2.

We have inductively defined two sequences $\left\{N^{(k)}\right\}_{k=0}^{\infty}$ and $\left\{B^{(k)}\right\}_{k=1}^{\infty}$ of non-negative integers. Obviously, $\left\{N^{(k)}\right\}_{k=0}^{\infty}$ is weakly decreasing, i.e., $N^{(0)}>N^{(1)} \geq \cdots N^{(k)} \geq \cdots$. We show that

$$
\forall k \in \mathbb{N}, \exists k^{\prime}>k \text { s.t. } N^{(k)}>N^{\left(k^{\prime}\right)} .
$$

Let $k \in \mathbb{N}$ be arbitrary. Let $K:=1+B^{(k+1)}$ and $k^{\prime}:=k+K$. We prove by contradiction that $N^{(k)}>N^{\left(k^{\prime}\right)}$. If $N^{(k)}=N^{(k+1)}=\cdots=N^{(k+K)}$, then by Claims k.2 to (k+K-1).2., we can conclude that $B^{(k+1)}>B^{(k+2)}>\cdots>B^{(k+K+1)}$. This implies $0>B^{(k+K+1)}$, a contradiction. Thus, (3) holds. However, $N^{(0)}$ is a finite non-negative number, a contradiction. This completes the proof of Theorem 1.

\section{Proof of Corollary 3}

Next we prove Corollary 3. We begin with three lemmas.

Lemma 5. Suppose that $\varphi$ is independent, and for each $t \in T, \Phi^{t}: \mathcal{P}\left(X^{t}\right)^{N^{t}} \rightarrow \mathcal{A}^{t}$ is strategy-proof and Pareto efficient. Suppose also that a strategy-proof rule $\zeta$ dominates $\varphi$. Then, for each $R \in \mathcal{D}$, if $\zeta(R)$ dom $\varphi(R)$, then $\zeta(R)$ does not coordinate-wise weakly dominate $\varphi(R)$ at $R$.

Proof. Suppose to the contrary that $\zeta(R) \mathrm{cw}-\operatorname{dom}(R) \varphi(R)$. Since $\zeta(R) \operatorname{dom}(R) \varphi(R)$, there exists $i \in N$ such that $\zeta_{i}(R) P_{i} \varphi_{i}(R)$. Since $R_{i}$ is separable, there exists $t \in T_{i}$ such that $\zeta_{i}^{t}(R) P_{i}^{t}$ $\varphi_{i}^{t}(R)=\Phi_{i}^{t}\left(R^{t}\right)$. Since $\zeta(R)$ cw-dom $(R) \varphi(R)$, for each $j \in N^{t} \backslash\{i\}, \zeta_{j}^{t}(R) R_{j}^{t} \varphi_{j}^{t}(R)=\Phi_{j}^{t}\left(R^{t}\right)$. This violates that $\Phi^{t}\left(R^{t}\right)$ is Pareto efficient at $R^{t}$.

Lemma 6. Suppose that $\varphi$ is independent and for each $t \in T, \Phi^{t}: \mathcal{P}\left(X^{t}\right)^{N^{t}} \rightarrow \mathcal{A}^{t}$ is strategy-proof and Pareto efficient. Suppose also that a strategy-proof rule $\zeta$ dominates $\varphi$. Let $R \in \mathcal{D}$ be such that $\zeta(R) \operatorname{dom}(R) \varphi(R)$. If $R$ does not satisfy the $(\zeta, \varphi)$-reverse property, then there exist $i \in N$ and $R_{i}^{\prime} \in \mathcal{D}_{i}$ such that 
(lem6-1) $R_{i} \notin \mathcal{P}\left(X_{i}\right)$

(lem6-2) $I\left(R_{i}^{\prime} ; R_{-i}\right)<I(R)$ and $\left(R_{i}^{\prime t}\right)_{t \in T_{i}}=\left(R_{i}^{t}\right)_{t \in T_{i}}$, and

(lem6-3) $\zeta\left(R_{i}^{\prime} ; R_{-i}\right) \operatorname{dom}\left(R_{i}^{\prime} ; R_{-i}\right) \varphi\left(R_{i}^{\prime} ; R_{-i}\right)$.

Proof. Same as the proof of Lemma 3.

Lemma 7. Suppose that a rule $\varphi$ is independent and for each $t \in T, \Phi^{t}: \mathcal{P}\left(X^{t}\right)^{N^{t}} \rightarrow \mathcal{A}^{t}$ is strategy-proof and Pareto efficient. Suppose also that a strategy-proof rule $\zeta$ dominates $\varphi$. Let $R^{(0)} \in \mathcal{D}$ be such that $\zeta\left(R^{(0)}\right) \operatorname{dom}\left(R^{0}\right) \varphi\left(R^{(0)}\right)$. If $R^{(0)}$ does not satisfy the $(\zeta, \varphi)$-reverse property, then there exists a finite sequence of agent-preference pairs $\left\{\left(j^{(\ell)}, R_{j^{(\ell)}}^{(\ell)}\right)_{\ell=1}^{L}\right.$ satisfying the following conditions (i), (ii) and (iii). For each $\ell=1, \cdots$, L, let $R^{(\ell)}:=\left(R_{j^{(\ell)}}^{(\ell)} ; R_{-j^{(\ell)}}^{(\ell-1)}\right)$.

(i) $\forall \ell=1, \cdots, L, R_{j^{(\ell)}}^{(\ell-1)} \notin \mathcal{P}\left(X_{j^{(\ell)}}\right)$,

(ii) $\forall \ell=1, \cdots, L,\left(R_{j^{(\ell)}}^{(\ell) t}\right)_{t \in T_{j}(\ell)}=\left(R_{j^{(\ell)}}^{(\ell-1) t}\right)_{t \in T_{j}(\ell)}$ and

(iii) $R^{(L)}$ satisfies the $(\zeta, \varphi)$-reverse property.

Proof. Same as the proof of Lemma 4.

We introduce notations: For each $i \in N$, each $t \in T_{i}$, each $R_{i}^{t} \in \mathcal{P}\left(X^{t}\right)$, each $R_{i} \in \mathcal{D}_{i}$ and each $R \in \mathcal{D}$, let $B^{\omega}\left(R_{i}^{t}\right):=\left|\operatorname{SUC}\left(R_{i}^{t}, \omega_{i}^{t}\right)\right|, B^{\omega}\left(R_{i}\right):=\sum_{t \in T_{i}} B^{\omega}\left(R_{i}^{t}\right)$, and $B^{\omega}(R):=\sum_{i \in N} B^{\omega}\left(R_{i}\right)$. The operator $B^{\omega}$ assigns the number of object(s) which are preferred to the endowed object(s). Now we are ready to prove Corollary 3 .

\section{Proof of Corollary 3}

Replacing $B$ in the proof of Theorem 1 with $B^{\omega}$, the same proof as that for Theorem 1 works, where for each $i \in N, \omega_{i}$ plays the role of $\left(o^{t}\right)_{t \in T_{i}}$ in the proof of Theorem 1 .

\section{References}

AbdulkadiroĞLu, A., P. A. Pathak, And A. E. Roth (2009): "Strategy-proofness versus Efficiency in Matching with Indifferences: Redesigning the NYC High School Match," American Economic Review, 99, 1954-1978.

AbdulkadiroĞLu, A., And T. Sönmez (1998): "Random Serial Dictatorship and the Core from Random Endowments in House Allocation Problems," Econometrica, 66, 689-701.

(1999): "House Allocation with Existing Tenants," Journal of Economic Theory, 88, 233-260.

(2003): "School Choice: A Mechanism Design Approach," Americal Economic Review, 93, 729-747. 
Anno, H. (2008): "Second Best Efficiency of Strategy-proof Allocation Rules and a Characterization of the Uniform Rule with Multiple Commodities," Working paper, Waseda university.

Anno, H., And H. SAsaki (2013): "Second-Best Efficiency of Allocation Rules: Strategyproofness and Single-peaked Preferences with Multiple Commodities," Economic Theory, 54, 693-716.

Balinski, M., And T. Sönmez (1999): "A Tale of Two Mechanisms: Student Placement," Journal of Economic Theory, 84, 73-94.

Barberà, S., J. Massó, And A. Neme (2005): "Voting by Committees under Constraints," Journal of Economic Theory, 122, 185-205.

Barberà, S., H. Sonnenschein, and L. Zhou (1991): "Voting by Committees," Econometrica, 59, 595-609.

Bloch, F., And D. Cantala (2013): "Markovian Assignment Rules," Social Choice and Welfare, $40,1-25$.

Bogomolnaia, A., And H. Moulin (2001): "A New Solution to the Random Assignment Problem," Journal of Economic Theory, 100, 295-328.

Budish, E., And E. Cantillon (2012): "The Multi-Unit Assignment Problem: Theory and Evidence from Course Allocation at Harvard," American Economic Review, 102(5), 2237-2271.

Che, Y.-K., And F. KoJima (2010): "Asymptotic Equivalence of Random Priority and Probabilistic Serial Mechainsms," Econometrica, 78, 1625-1672.

Chen, P., M. Egesdal, M. Pycia, and M. B. Yenmez (2014): "Ranking by manipulability and quantile stable mechanisms," Working paper.

Dur, U. (2012): "Dynamic School Choice Problem," Working paper.

Dur, U., And T. Wiseman (2015): "School Choice with Neighbors," Working paper.

ERDIL, A. (2014): "Strategy-proof Stochastic Assignment," Journal of Economic Theory, 151, $146-162$.

Erdil, A., And H. Ergin (2007): "Two-Sided Matching with Indifferences," University of Oxford and Washington University, unpublished mimeo.

Ergin, H. (2002): "Efficient Resource Allocation on the Basis of Priorities," Econometrica, 70, 2489-2498.

Ergin, H., T. Sönmez, And M. U. Ünver (2014): "Lung Exchange," Working paper.

Fishburn, P. C. (1970): Utility Theory for Decision Making. John Wiley \& Sons, New York. 
Fragiadakis, D., And P. Troyan (2013): "Market Design under Distributional Constraints: Diversity in School Choice and Other Applications," Working paper.

Gale, D., And L. S. Shapley (1962): "College Admissions and the Stability of Marriage," American Mathematical Monthly, 69, 9-15.

Hammond, P. J. (2000): "Reassessing the Diamond-Mirrlees Efficiency Theorem," in Incentives, Organization, and Public Economics: Papers in Honour of Sir James Mirrlees, ed. by P. J. Hammond, and G. D. Myles, pp. 193-216.

Hatfield, J. W. (2009): "Strategy-proof, Efficient, and Nonbossy Quota Allocations," Social Choice and Welfare, 33, 505-515.

Hylland, A., and R. Zeckhauser (1979): "The Efficient Allocation of Individuals to Positions," Journal of Political Economy, 87, 293-314.

Ju, B.-G. (2003): "A Characterization of Strategy-proof Voting Rules for Separable Weak Orderings," Social Choice and Welfare, 21, 469-499.

Kennes, J., D. Monte, and N. Tumennasan (2014): "The Daycare Assignment: A Dynamic Matching Problem," American Economic Journal: Microeconomics, 6(4), 362-406.

Kesten, O. (2006): "On Two Competing Mechanisms for Priority-based Allocation Problems," Journal of Economic Theory, 127, 155-171.

(2010): "School Choice with Consent," Quaterly Journal of Economics, 125(3), 12971348.

Kesten, O., And M. Kurino (2013): "Do Outside Options Matter in School Choice? A New Perspective on the Efficiency vs. Strategy-proofness Trade-off," Working paper.

Klaus, B. (2008): "The Coodinate-Wise Core for Multiple-Type Housing Markets Is Second-Best Incentive Compatible," Journal of Mathematical Economics, 44, 919-924.

Klaus, B., And E. Miyagawa (2001): "Strategy-proofness, Solidarity, and Consistency for Multiple Assignment Problems," International Journal of Game Theory, 30, 421-435.

Konishi, H., T. Quint, And J. Wako (2001): "On the Shapley-Scarf Economy: The Case of Multiple Types of Indivisible Goods," Journal of Mathematical Economics, 35, 1-15.

Kurino, M. (2014): "House Allocation with Overlapping Generations," American Economic Journal: Microeconomics, 6 (1), 258-289.

Le Breton, M., And A. Sen (1999): "Separable Preferences, Strategyproofness, and Decomposability," Econometrica, 67, 605-628.

Monte, D., And N. Tumennasan (2013): "Centralized Allocation in Multiple Markets," Working Paper. 
Moulin, H. (1995): Cooperative Microeconomics: A Game-Theoretic Introduction. Princeton University Press.

PÁPAI, S. (2000): "Strategyproof Assignment by Hierarchical Exchange," Econometrica, 68, 14031433.

(2001): "Strategyproof and Nonbossy Multiple Assignments," Journal of Public Economic Theory, 3, 257-271.

Pathak, P. A., And T. Sönmez (2013): "School Admissions Reform in Chicago and England: Comparing Mechanisms by Their Vulnerability to Manipulation," American Economic Review, 103(1), 80-106.

Pereyra, J. S. (2013): "A Dynamic School Choice Model," Games and Economic Behavior, 80, $100-114$.

Pycia, M., AND M. U. Ünver (2009): "Incentive Compatible Allocation and Exchange of Discrete Resources," Boston College Working Papers in Economics 715.

Roth, A. E., And A. Postlewaite (1977): "Weak Versus Strong Domination in a Market with Indivisible Goods," Journal of Mathematical Economics, 4, 131-137.

Roth, A. E., T. Sönmez, And M. U. ÜnVer (2004): "Kidney Exchange," Quarterly Journal of Economics, 119, 457-488.

(2005): "Pairwise Kidney Exchange," Journal of Economic Theory, 125, 151-188.

SAsaki, H. (2003): "Limitation of Efficiency: Strategy-proofness and Single-peaked Preferences with Many Commodities," Working Paper.

Shapley, L., And H. Scarf (1974): "On Cores and Indivisibility," Journal of Mathematical Economics, 1, 23-37.

Sönmez, T., And T. B. Switzer (2013): "Matching with (Branch-of-Choice) Contracts at the United States Military Academy," Econometrica, 81, 451-488-185.

Sönmez, T., And M. U. Ünver (2010): "Course Bidding at Business Schools," International Economic Review, 51, 99-123.

(2013): "Market Design for Kidney Exchange," in The Handbook of Market Design, ed. by M. N. Zvika Neeman, and N. Vulkan, pp. 93-137. Oxford University Press, Oxford.

Sönmez, T., And U. Ünver (2011): "Matching, Allocation, and Exchange of Discrete Resources," in Handbook of Social Economics, ed. by J. Benhabib, A. Bisin, and M. O. Jackson, vol. 1A, pp. 781-852. North Holland, The Netherlands.

The Japan Society for Transplantation (2013): Fact Book (in Japanese). 
Whitehead, C., And K. J. Scanlon (2007): Social Housing in Europe. LSE London, Chapter 2.

YILmaz, Özgür. (2011): "Kidney Exchange: An Egalitarian Mechanism," Journal of Economic Theory, 146, 592-618. 